\title{
LOCALLY COMPACT QUANTUM GROUPS IN THE VON NEUMANN ALGEBRAIC SETTING
}

\author{
JOHAN KUSTERMANS* and STEFAAN VAES**
}

\begin{abstract}
In this paper we complete in several aspects the picture of locally compact quantum groups. First of all we give a definition of a locally compact quantum group in the von Neumann algebraic setting and show how to deduce from it a $\mathrm{C}^{*}$-algebraic quantum group. Further we prove several results about locally compact quantum groups which are important for applications, but were not yet settled in our paper [8]. We prove a serious strengthening of the left invariance of the Haar weight, and we give several formulas connecting the locally compact quantum group with its dual. Loosely speaking we show how the antipode of the locally compact quantum group determines the modular group and modular conjugation of the dual locally compact quantum group.
\end{abstract}

\section{Introduction}

Building on the work of Kac and Vainerman [15], Enock and Schwartz [3], Baaj and Skandalis [1], Woronowicz [18] and Van Daele [16] a precise definition of a locally compact quantum group was recently introduced by the authors in [8], see [9] and [10] for an overview. For an overview of the historic development of the theory we refer to [10] and the introduction to [8]. Because commutative $\mathrm{C}^{*}$-algebras are always of the form $C_{0}(X)$, where $X$ is a locally compact space and $C_{0}(X)$ denotes the $\mathrm{C}^{*}$-algebra of continuous functions on $X$ vanishing at infinity, arbitrary $\mathrm{C}^{*}$-algebras are sometimes thought of as the algebra of continuous functions vanishing at infinity on a (non-existing) locally compact quantum space. For this reason the $\mathrm{C}^{*}$-algebra framework is the most natural one to study locally compact quantum groups. The most general commutative example of a locally compact quantum group is $C_{0}(G)$ with comultiplication $\Delta: C_{0}(G) \rightarrow C_{b}(G \times G)$ given by $(\Delta f)(x, y)=f(x y)$, where $G$ is a locally compact group and $C_{b}$ denotes the algebra of continuous bounded functions. This philosophy is followed in [8] where we defined 'reduced $\mathrm{C}^{*}$ algebraic quantum groups' as the proper notion of a locally compact quantum

\footnotetext{
* The first author is a Post-doctoral Researcher of the Fund for Scientific Research Flanders Belgium (F.W.O.)

** The second author is a Research Assistent of the Fund for Scientific Research Flanders Belgium (F.W.O.)

Received May 25, 2000; in revised form November 15, 2000.
} 
group in the $\mathrm{C}^{*}$-algebra framework. As already explained, the most general commutative example is $C_{0}(G)$ where $G$ is a locally compact group. Further the theory unifies compact quantum groups and Kac algebras and it includes known examples as the quantum Heisenberg group, quantum $E$ (2)-group, quantum Lorentz group and quantum $a z+b$-group. Within this theory one can construct a dual reduced $\mathrm{C}^{*}$-algebraic quantum group and prove a Pontryagin duality theorem.

On a technical level it is often more easy to work with von Neumann algebras rather than $\mathrm{C}^{*}$-algebras, certainly when dealing with weights. So, already in [8], we associated with every reduced $\mathrm{C}^{*}$-algebraic quantum group a von Neumann algebraic quantum group and we used it to prove several results on the $\mathrm{C}^{*}$-algebra level. The first aim of this paper is to give an intrinsic definition of a von Neumann algebraic quantum group and to associate with it, in a canonical way, a reduced $\mathrm{C}^{*}$-algebraic quantum group. This can be thought of as the quantum analogue of the classical result of Weil (see [17, Appendice I]), stating that every group with an invariant measure has a unique topology turning it into a locally compact group.

A second aim of this paper is to prove some new results on both $\mathrm{C}^{*}$-algebraic and von Neumann algebraic quantum groups, which are indispensable for applications. In our definition of either $\mathrm{C}^{*}$-algebraic or von Neumann algebraic quantum groups we assume the existence of left and right invariant weights. But the property of invariance we assume is quite weak, and in this paper we show how a much stronger notion of invariance can be proved. The same kind of result is stated for Kac algebras in [2], but not proved. The first proof was given by Zsidó in [19] (see also remark 18.23 in [12]). This stronger invariance property is needed whenever an action of a von Neumann algebraic quantum group on a von Neumann algebra appears: see [5] and [13], but also [2] for Kac algebra actions, and it will certainly be useful in future investigations as well.

Further we will complete the picture of the quantum group and its dual with several formulas giving a link between the antipode of the quantum group and the modular theory of its dual. Roughly speaking we obtain that

$$
\hat{T}^{*} \Lambda(x)=\Lambda\left(S\left(x^{*}\right)\right)
$$

for nice $x \in M$, where $M$ is the von Neumann algebraic quantum group, $\Lambda$ is the GNS-map of the left invariant weight $\varphi$ on $M, S$ is the antipode and $\hat{T}$ is the operator appearing in the modular theory of the left invariant weight $\hat{\varphi}$ on the dual von Neumann algebraic quantum group: it is the closure of $\hat{\Lambda}(\omega) \mapsto \hat{\Lambda}\left(\omega^{*}\right)$ where $\hat{\Lambda}$ is the GNS-map of $\hat{\varphi}$. To these results and formulas will be referred in further research, see e.g. [5] and [13]. 
We end this introduction with some conventions and references. We assume the reader to be familiar with the theory of normal semi-finite faithful weights (in short, n.s.f. weights) on von Neumann algebras, but let us fix some notations. So let $\varphi$ be a n.s.f. weight on a von Neumann algebra $M$. Then we define the following sets: (1) $\mathscr{M}_{\varphi}^{+}=\left\{x \in M^{+} \mid \varphi(x)<\infty\right\}$, (2) $\mathscr{N}_{\varphi}=\{x \in M \mid$ $\left.x^{*} x \in \mathscr{M}_{\varphi}^{+}\right\}$and (3) $\mathscr{M}_{\varphi}=$ the linear span of $\mathscr{M}_{\varphi}^{+}$in $M$. There exists a unique linear map $F: \mathscr{M}_{\varphi} \rightarrow \mathrm{C}$, extending $\varphi$. For all $x \in \mathscr{M}_{\varphi}$, we set $\varphi(x)=$ $F(x)$. We refer to definition 1.2 of [8] for the definition of a GNS-construction $\left(H_{\varphi}, \pi_{\varphi}, \Lambda_{\varphi}\right)$ for $\varphi$. As usual we introduce the closed linear operator $T$ in $H_{\varphi}$ as the closure of the map $\Lambda_{\varphi}(x) \mapsto \Lambda_{\varphi}\left(x^{*}\right)$ for $x \in \mathscr{N}_{\varphi} \cap \mathscr{N}_{\varphi}^{*}$. Making the polar decomposition $T=J \nabla^{\frac{1}{2}}$ of $T$, we obtain the modular operator $\nabla$ and modular conjugation $J$ of $\varphi$ in the GNS-construction $\left(H_{\varphi}, \pi_{\varphi}, \Lambda_{\varphi}\right)$.

For the definition of the extended positive part $M_{\mathrm{Ext}}^{+}$and operator valued weights, we refer to [4]. For $T \in M_{\mathrm{Ext}}^{+}$and $\omega \in M_{*}^{+}$, we set $\langle T, \omega\rangle=T(\omega) \in$ $[0, \infty]$. Recall that $M^{+}$is naturally embedded in $M_{\mathrm{Ext}}^{+}$.

Consider another von Neumann algebra $N$. Let $\psi$ be a n.s.f. weight on $N$ with GNS-construction $\left(H_{\psi}, \pi_{\psi}, \Lambda_{\psi}\right)$. The tensor product weight $\varphi \otimes \psi$ is a n.s.f. weight on $M \otimes N$ (see e.g. definition 8.2 of [12]) that has a GNSconstruction $\left(H_{\varphi} \otimes H_{\psi}, \pi_{\varphi} \otimes \pi_{\psi}, \Lambda_{\varphi} \otimes \Lambda_{\psi}\right)$, where $\Lambda_{\varphi} \otimes \Lambda_{\psi}$ is the $\sigma$-strong* closure of the algebraic tensor product $\Lambda_{\varphi} \odot \Lambda_{\psi}: \mathscr{N}_{\varphi} \odot \mathscr{N}_{\psi} \rightarrow H_{\varphi} \otimes H_{\psi}$.

Identifying $N$ with $\mathrm{C} \otimes N$ as a von Neumann subalgebra of $M \otimes N$, we may consider operator valued weights from $M \otimes N$ to $N$. Define the operator valued weight $\varphi \otimes \iota:(M \otimes N)^{+} \rightarrow N_{\text {Ext }}^{+}$such that $\langle(\varphi \otimes \iota)(x), \omega\rangle=\varphi((\iota \otimes \omega)(x))$ for $x \in(M \otimes N)^{+}, \omega \in N_{*}^{+}$.

When $L$ is some set of elements of a space we denote by $\langle L\rangle$ the linear span of $L$ and by $[L]$ the closed linear span. The symbol $\otimes$ will denote either a von Neumann algebraic tensor product or a tensor product of Hilbert spaces and $\iota$ will denote the identity map. Finally we use the symbol $\chi$ to denote the flip map from $M \otimes N$ to $N \otimes M$, where $N$ and $M$ are von Neumann algebras. We use $\Sigma$ to denote the flip map from $H \otimes K$ to $K \otimes H$ when $H$ and $K$ are Hilbert spaces.

\section{Von Neumann algebraic quantum groups}

We state the definition of a von Neumann algebraic quantum group and discuss how the $\mathrm{C}^{*}$-algebraic theory can be translated to the von Neumann algebraic setting. The major difference between both approaches is the absence of density conditions in the definition of von Neumann algebraic quantum groups: these will follow automatically!

Definition 1.1. Consider a von Neumann algebra $M$ together with a unital normal ${ }^{*}$-homomorphism $\Delta: M \rightarrow M \otimes M$ such that $(\Delta \otimes \iota) \Delta=(\iota \otimes \Delta) \Delta$. 
Assume moreover the existence of

(1) a n.s.f. weight $\varphi$ on $M$ that is left invariant: $\varphi((\omega \otimes \iota) \Delta(x))=\varphi(x) \omega(1)$ for all $\omega \in M_{*}^{+}$and $x \in \mathscr{M}_{\varphi}^{+}$.

(2) a n.s.f. weight $\psi$ on $M$ that is right invariant: $\psi((\iota \otimes \omega) \Delta(x))=$ $\psi(x) \omega(1)$ for all $\omega \in M_{*}^{+}$and $x \in \mathscr{M}_{\psi}^{+}$.

Then we call the pair $(M, \Delta)$ a von Neumann algebraic quantum group.

In the next part of this section we will introduce the objects essential to these quantum groups. Most of the time the results and proofs in [8] can be easily translated to the von Neumann algebraic setting by replacing the norm and strict topology in the considerations by the $\sigma$-strong* topology. However, some care has to be taken to prove the density conditions and we will do this in detail.

For the rest of this paper we fix a von Neumann algebraic quantum group $(M, \Delta)$, assuming that $M$ is in standard form with respect to a Hilbert space $H$. At the same time we fix a n.s.f. left invariant weight $\varphi$ on $(M, \Delta)$ together with a GNS-construction $(H, \iota, \Lambda)$. We also choose a n.s.f. right invariant weight $\psi$ on $(M, \Delta)$ together with a GNS-construction $(H, \iota, \Gamma)$ (later on we will make some canonical choices for $\psi$ and $\Gamma$ ). Denote the modular group of $\varphi$ by $\sigma$. We let $\nabla$ denote the modular operator and $J$ the modular conjugation of $\varphi$ with respect to the GNS-construction $(H, \iota, \Lambda)$.

By left invariance of $\varphi$, we get that $(\omega \otimes \imath) \Delta(x) \in \mathscr{N}_{\varphi}$ for all $x \in \mathscr{N}_{\varphi}$ and $\omega \in M_{*}$. Arguing as in result 2.6 of [8], we also get for $a, b \in \mathscr{N}_{\psi}$ and $x \in \mathscr{N}_{\varphi}$ that $(\psi \otimes \iota)\left(\Delta\left(b^{*} x\right)(a \otimes 1)\right) \in \mathscr{N}_{\varphi}$. Along the way to the proof of theorem 1.2, one also translates proposition 3.15 of [8], giving rise to the important equalities

$$
\begin{aligned}
H & =\left[\Lambda((\omega \otimes \iota) \Delta(x)) \mid x \in \mathscr{N}_{\varphi}, \omega \in M_{*}\right] \\
& =\left[\Lambda\left((\psi \otimes \iota)\left(\Delta\left(b^{*} x\right)(a \otimes 1)\right)\right) \mid x \in \mathscr{N}_{\varphi}, a, b \in \mathscr{N}_{\psi}\right] .
\end{aligned}
$$

The left invariance of $\varphi$ implies that $\Delta(y)(x \otimes 1) \in \mathscr{N}_{\varphi \otimes \varphi}$ for all $x, y \in \mathscr{N}_{\varphi}$. Copying the proof of theorem 3.16 of [8], we get moreover the following result.

THEOREM 1.2. There exists a unique unitary element $W \in B(H \otimes H)$ such that $W^{*}(\Lambda(x) \otimes \Lambda(y))=(\Lambda \otimes \Lambda)(\Delta(y)(x \otimes 1))$ for all $x, y \in \mathscr{N}_{\varphi}$.

Since $(\omega \otimes \iota)\left(W^{*}\right) \Lambda(x)=\Lambda((\omega \otimes \iota) \Delta(x))$ for all $x \in \mathscr{N}_{\varphi}$ and $\omega \in B(H)_{*}$ (see result 2.10 of [8]), the commutant theorem for the tensor product of von Neumann algebras implies that $W \in M \otimes B(H)$.

One checks that $\Delta(x)=W^{*}(1 \otimes x) W$ for all $x \in M$ and that $W_{12} W_{13} W_{23}=$ $W_{23} W_{12}$ (see proposition 3.18 of [8]). We call $W$ the multiplicative unitary of $(M, \Delta)$ with respect to the GNS-construction $(H, \iota, \Lambda)$. 
It goes without saying that all these results also have their right invariant counterparts. For later purposes we introduce the unitary element $V \in B(H) \otimes$ $M$ such that $V(\Gamma(x) \otimes \Gamma(y))=(\Gamma \otimes \Gamma)(\Delta(x)(1 \otimes y))$ for all $x, y \in \mathscr{N}_{\psi}$. As in result 2.10 of [8], one proves that
(3)
$\left(\omega_{\Gamma(a), \Gamma(b)} \otimes \iota\right)\left(V^{*}\right)=(\psi \otimes \iota)\left(\Delta\left(b^{*}\right)(a \otimes 1)\right)$
for all $a, b \in \mathscr{N}_{\psi}$.

The proof of proposition 3.22 of [8] survives the translation to the von Neumann algebra setting. Combining this with equation (2) we arrive at the following conclusion.

Proposition 1.3. There exists a unique densely defined closed antilinear operator $G$ in $H$ such that $\left\langle\Lambda\left((\psi \otimes \iota)\left(\Delta\left(x^{*}\right)(y \otimes 1)\right)\right) \mid x, y \in \mathscr{N}_{\varphi}^{*} \mathscr{N}_{\psi}\right\rangle$ is a core for $G$ and

$$
G \Lambda\left((\psi \otimes \iota)\left(\Delta\left(x^{*}\right)(y \otimes 1)\right)\right)=\Lambda\left((\psi \otimes \iota)\left(\Delta\left(y^{*}\right)(x \otimes 1)\right)\right)
$$

for $x, y \in \mathscr{N}_{\varphi}^{*} \mathscr{N}_{\psi}$. Take the polar decomposition $G=I N^{\frac{1}{2}}$ of $G$ to get a strictly positive operator $N$ in $H$ and an anti-unitary operator $I$ on $H$. Then $I=I^{*}, I^{2}=1$ and $I N I=N^{-1}$.

A careful analysis of the proof of proposition 5.5 of [8] reveals that it remains true in the present setting. Using equation (3), the techniques in the proof of proposition 5.8 of [8], and appealing to the proof of result 5.10 of [8], one arrives at the commutation relation

$$
V\left(\nabla_{\psi} \otimes N\right)=\left(\nabla_{\psi} \otimes N\right),
$$

where $\nabla_{\psi}$ denotes the modular operator of $\psi$ with respect to the GNS-construction $(H, \iota, \Gamma)$.

Up till now, we did not need the density conditions that are present in the definition of reduced $\mathrm{C}^{*}$-algebraic quantum groups (see definition 4.1 of [8]). This is the case because we were only working on the Hilbert space level for which the relevant density conditions are already established in equations (1) and (2). In order to further develop the theory along the lines of [8], we will now prove similar density conditions on the level of the von Neumann algebra $M$. The idea of the proof is taken from $[3,2.7 .6]$.

Proposition 1.4. Denoting by ${ }^{-}$the $\sigma$-strong* closure we have

$$
\begin{aligned}
M & =\left\langle(\omega \otimes \imath) \Delta(x) \mid x \in M, \omega \in M_{*}\right\rangle^{-} \\
& =\left\langle(\iota \otimes \omega) \Delta(x) \mid x \in M, \omega \in M_{*}\right\rangle^{-} \\
& =\left\{(\omega \otimes \iota)(V) \mid \omega \in B(H)_{*}\right\}^{-} .
\end{aligned}
$$


Proof. Define $\mathscr{T}_{\psi}$ to be the Tomita ${ }^{*}$-algebra of $\psi$. From formula (3) it follows that

$$
\begin{aligned}
\left\{(\omega \otimes \iota)(V) \mid \omega \in B(H)_{*}\right\}^{-} \\
\quad=\left\langle(\psi \otimes \iota)\left(\left(c a^{*} \otimes 1\right) \Delta(b)\right) \mid a, b \in \mathscr{N}_{\psi}, c \in \mathscr{T}_{\psi}\right\rangle^{-} \\
\quad=\left\langle(\psi \otimes \iota)\left(\left(a^{*} \otimes 1\right) \Delta(b)\left(\sigma_{-i}^{\psi}(c) \otimes 1\right)\right) \mid a, b \in \mathscr{N}_{\psi}, c \in \mathscr{T}_{\psi}\right\rangle^{-} \\
\quad=\left\langle(\omega \otimes \iota) \Delta(x) \mid x \in M, \omega \in M_{*}\right\rangle^{-} .
\end{aligned}
$$

Now we define

$$
M_{r}=\left\langle(\omega \otimes \iota) \Delta(x) \mid x \in M, \omega \in M_{*}\right\rangle^{-} .
$$

Because $V$ is a multiplicative unitary the linear space $\{(\omega \otimes \iota)(V) \mid \omega \in$ $\left.B(H)_{*}\right\}$ is an algebra that acts non-degenerately on $H$. Because $M_{r}$ is clearly self-adjoint, we get that $M_{r}$ is a von Neumann subalgebra of $M$. Working with the von Neumann algebraic quantum group $(M, \chi \Delta)$ instead of $(M, \Delta)$ we obtain that also

$$
M_{l}=\left\langle(\iota \otimes \omega) \Delta(x) \mid x \in M, \omega \in M_{*}\right\rangle^{-}
$$

is a von Neumann subalgebra of $M$. Observe that it follows from the commutant theorem for the tensor product of von Neumann algebras that $\Delta(x) \in M_{l} \otimes M_{r}$ for all $x \in M$.

Then we conclude from equation (4) that it is possible to define a oneparameter group $\left(\tau_{t}\right)_{t \in \mathrm{R}}$ of automorphisms of $M_{r}$ by $\tau_{t}(x)=N^{-i t} x N^{i t}$ for all $x \in M_{r}$ and $t \in \mathrm{R}$. It also follows from equation (4) and the fact $\Delta(x)=$ $V(x \otimes 1) V^{*}$ for all $x \in M$, that we have $\Delta\left(\sigma_{t}^{\psi}(x)\right)=\left(\sigma_{t}^{\psi} \otimes \tau_{-t}\right) \Delta(x)$ for all $x \in M$ and $t \in \mathrm{R}$, which makes sense because $\Delta(x) \in M \otimes M_{r}$. For the same reason we can write

$$
M_{l}=\left\{(\iota \otimes \omega) \Delta(x) \mid x \in M, \omega \in\left(M_{r}\right)_{*}\right\}^{-}
$$

and because $\sigma_{t}^{\psi}((\iota \otimes \omega) \Delta(x))=\left(\iota \otimes \omega \tau_{t}\right) \Delta\left(\sigma_{t}^{\psi}(x)\right)$ for all $\omega \in\left(M_{r}\right)_{*}$ and $x \in M$, we get $\sigma_{t}^{\psi}\left(M_{l}\right)=M_{l}$ for all $t \in \mathrm{R}$. By the right invariance of $\psi$ it follows that the restriction $\psi_{l}$ of $\psi$ to $M_{l}$ is semifinite. By Takesaki's theorem (see e.g. $[12,10.1]$ ) there exists a unique normal faithful conditional expectation $E$ from $M$ to $M_{l}$ satisfying $\psi(x)=\psi_{l}(E(x))$ for all $x \in M^{+}$. From $[12,10.2]$ it follows that $E(x) P=P x P$ for all $x \in M$, where $P$ denotes the orthogonal projection onto the closure of $\Gamma\left(\mathscr{N}_{\psi} \cap M_{l}\right)$. So the range of $P$ contains $\Gamma((\iota \otimes \omega) \Delta(x))$ for all $\omega \in M_{*}$ and $x \in \mathscr{N}_{\psi}$. By the right invariant 
version of equation (1) we get that $P=1$. So $E$ is the identity map and $M_{l}=M$.

Working with the von Neumann algebraic quantum group $(M, \chi \Delta)$ we obtain $M=M_{r}$. We already proved that $M_{r}$ is the $\sigma$-strong* closure of $\{(\omega \otimes$ $\left.\iota)(V) \mid \omega \in B(H)_{*}\right\}$ and so this concludes the proof of the proposition.

In the next part of this section, we introduce the most important objects associated to $(M, \Delta)$. For their properties, we refer to [8].

There exist a unique *anti-automorphism $R$ on $M$ and a unique $\sigma$-strongly* continuous one parameter group $\tau$ on $M$ such that $R(x)=I x^{*} I$ and $\tau_{t}(x)=$ $N^{-i t} x N^{i t}$ for all $x \in M$ and $t \in \mathrm{R}$. Then $\tau$ and $R$ commute and we define $S=R \tau_{-\frac{i}{2}}$. We call $S$ the antipode, $R$ the unitary antipode and $\tau$ the scaling group of our quantum group $(M, \Delta)$. The space $\left\langle(\iota \otimes \varphi)\left(\Delta\left(a^{*}\right)(1 \otimes b)\right)\right|$ $\left.a, b \in \mathscr{N}_{\varphi}\right\rangle$ is a $\sigma$-strong* core for $S$ and

(5) $S\left((\iota \otimes \varphi)\left(\Delta\left(a^{*}\right)(1 \otimes b)\right)\right)=(\iota \otimes \varphi)\left(\left(1 \otimes a^{*}\right) \Delta(b)\right)$ for all $a, b \in \mathscr{N}_{\varphi}$.

There exists a positive number $v>0$ satisfying $\varphi \tau_{t}=v^{-t} \varphi$ for all $t \in \mathbf{R}$. The number $v$ is referred to as the scaling constant of $(M, \Delta)$. Use this relative invariance to define the strictly positive operator $P$ in $H$ such that $P^{i t} \Lambda(x)=$ $v^{\frac{t}{2}} \Lambda\left(\tau_{t}(x)\right)$ for all $t \in \mathrm{R}$ and $x \in \mathscr{N}_{\varphi}$.

Since $\chi(R \otimes R) \Delta=\Delta R$, we may and will choose $\psi$ to be equal to $\varphi R$ from now on. The modular group of $\psi$ will be denoted by $\sigma^{\prime}$. We have that $\psi \sigma_{t}=v^{-t} \psi$ for $t \in \mathrm{R}$. Hence there exists a positive self-adjoint operator $\delta$ affiliated to $M$ such that $\sigma_{t}(\delta)=v^{t} \delta$ for all $t \in \mathrm{R}$ and $\psi=\varphi_{\delta}$ (see definition 1.3 of [14]). We call $\delta$ the modular element of $(M, \Delta)$. Now we choose the GNS-construction $(H, \iota, \Gamma)$ for $\psi$ such that $\Gamma=\Lambda_{\delta}$ (see the remarks before proposition 1.15 in [8]).

\subsection{The dual von Neumann algebraic quantum group}

Following chapter 3 of [3], we introduce the dual von Neumann algebraic quantum group and its Haar weights. (see section 8 of [8]). The Banach space $M_{*}$ is a Banach algebra for the product defined by $\omega \theta=(\omega \otimes \theta) \Delta$ for all $\omega, \theta \in M_{*}$. Let $\lambda: M_{*} \rightarrow B(H)$ denote the injective algebra homomorphism such that $\lambda(\omega)=(\omega \otimes \iota)(W)$ for all $\omega \in M_{*}$.

Definition 1.5. Define $\hat{M}$ to be the $\sigma$-strong* closure of the algebra $\left\{\lambda(\omega) \mid \omega \in B(H)_{*}\right\}$. Then $\hat{M}$ is a von Neumann algebra and there exists a unique unital normal ${ }^{*}$-homomorphism $\hat{\Delta}: \hat{M} \rightarrow \hat{M} \otimes \hat{M}$ such that $\hat{\Delta}(x)=\Sigma W(x \otimes 1) W^{*} \Sigma$ for all $x \in \hat{M}$. The pair $(\hat{M}, \hat{\Delta})$ is again a von Neumann algebraic quantum group, referred to as the dual of $(M, \Delta)$. 
Let us recall the construction of the dual weight $\hat{\varphi}$. First of all, we define

$$
\mathscr{I}=\left\{\omega \in M_{*}\left|\exists M \in \mathrm{R}^{+}:\right| \omega\left(x^{*}\right) \mid \leq M\|\Lambda(x)\| \text { for all } x \in \mathscr{N}_{\varphi}\right\} .
$$

By Riesz' theorem, there exists for every $\omega \in \mathscr{I}$ a unique element $\xi(\omega) \in H$ such that $\omega\left(x^{*}\right)=\langle\xi(\omega), \Lambda(x)\rangle$ for all $x \in \mathscr{N}_{\varphi}$. The dual weight $\hat{\varphi}$ is by definition the unique n.s.f. weight on $\hat{M}$ having a GNS-construction $(H, \iota, \hat{\Lambda})$ such that $\lambda(\mathscr{I})$ is a $\sigma$-strong*-norm core for $\hat{\Lambda}$ and $\hat{\Lambda}(\lambda(\omega))=\xi(\omega)$ for all $\omega \in \mathscr{I}$. We denote the modular group of $\hat{\varphi}$ by $\hat{\sigma}$. The weight $\hat{\varphi}$ is left invariant with respect to $(\hat{M}, \hat{\Delta})$.

We denote the antipode, unitary antipode and scaling group of $(\hat{M}, \hat{\Delta})$ by $\hat{S}, \hat{R}$ and $\hat{\tau}$ respectively. The scaling constant of $(\hat{M}, \hat{\Delta})$ is equal to $v^{-1}$. Define the right invariant n.s.f. weight $\hat{\psi}$ on $(\hat{M}, \hat{\Delta})$ as $\hat{\psi}=\hat{\varphi} \hat{R}$. The modular group of $\hat{\psi}$ will be denoted by $\hat{\sigma}^{\prime}$. Denote the modular element of $(\hat{M}, \hat{\Delta})$ by $\hat{\delta}$. Referring to the fact that $\hat{\psi}=\hat{\varphi}_{\hat{\delta}}$, we define the GNS-construction $(H, \iota, \hat{\Gamma})$ of $\hat{\psi}$ such that $\hat{\Gamma}=\hat{\Lambda}_{\hat{\delta}}$.

The modular operator and modular conjugation of $\hat{\varphi}$ with respect to $(H, \iota, \hat{\Lambda})$ will be denoted by $\hat{\nabla}$ and $\hat{J}$ respectively. It is also worth mentioning that $P^{i t} \hat{\Lambda}(x)=v^{-\frac{t}{2}} \hat{\Lambda}\left(\hat{\tau}_{t}(x)\right)$ for all $t \in \mathrm{R}$ and $x \in \mathscr{N}_{\hat{\varphi}}$.

We can also construct the dual $(\hat{\hat{M}}, \hat{\hat{\Delta}})$ of $(\hat{M}, \hat{\Delta})$. If we use the GNSconstruction $(H, \iota, \hat{\Lambda})$ for the construction of the dual $(\hat{\hat{M}}, \hat{\hat{\Delta}})$, the Pontryagin duality theorem says that $(\hat{\hat{M}}, \hat{\hat{\Delta}})=(M, \Delta)$. We even have that $\hat{\hat{\varphi}}=\varphi$ and $\hat{\hat{\Lambda}}=\Lambda$. Since $(\hat{\hat{M}}, \hat{\hat{\Delta}})=(M, \Delta)$, we get that $\hat{\hat{\delta}}=\delta$. Hence $\hat{\hat{\Gamma}}=\Lambda_{\delta}=\Gamma$.

\subsection{From von Neumann algebraic to $\mathrm{C}^{*}$-algebraic quantum groups}

In [8], we associated to any reduced $\mathrm{C}^{*}$-algebraic quantum group a von Neumann algebraic quantum group by taking the $\sigma$-strong* closure of the underlying $\mathrm{C}^{*}$-algebra in the GNS-space of a left Haar weight. In the last part of this section we go the other way around by introducing a $\mathrm{C}^{*}$-algebraic quantum group.

To distinguish between von Neumann algebraic and $\mathrm{C}^{*}$-algebraic tensor products we will denote the minimal $\mathrm{C}^{*}$-tensor product by $\otimes_{\mathrm{c}}$.

Proposition 1.6. Define $M_{\mathrm{c}}$ to be the norm closure of the space $\{(\iota \otimes$ $\left.\omega)(W) \mid \omega \in B(H)_{*}\right\}$ and $\Delta_{\mathrm{c}}$ to be the restriction of $\Delta$ to $M_{\mathrm{c}}$. Then the pair $\left(M_{\mathrm{c}}, \Delta_{\mathrm{c}}\right)$ is a reduced $\mathrm{C}^{*}$-algebraic quantum group.

Proof. Because $W$ is manageable and $\Delta_{\mathrm{c}}(x)=W^{*}(1 \otimes x) W$ for all $x \in$ $M_{\mathrm{c}}$, propositions 1.5 and 5.1 of [18] imply that $M_{\mathrm{c}}$ is a $\mathrm{C}^{*}$-algebra, $\Delta_{\mathrm{c}}$ is a non-degenerate ${ }^{*}$-homomorphism from $M_{\mathrm{c}}$ into the multiplier algebra of 
$M_{\mathrm{c}} \otimes_{\mathrm{c}} M_{\mathrm{c}}$, such that $\left(\Delta_{\mathrm{c}} \otimes_{\mathrm{c}} \iota\right) \Delta_{\mathrm{c}}=\left(\iota \otimes_{\mathrm{c}} \Delta_{\mathrm{c}}\right) \Delta_{\mathrm{c}}$ and both $\Delta_{\mathrm{c}}\left(M_{\mathrm{c}}\right)\left(M_{\mathrm{c}} \otimes 1\right)$ and $\Delta_{\mathrm{c}}\left(M_{\mathrm{c}}\right)\left(1 \otimes M_{\mathrm{c}}\right)$ are dense in $M_{\mathrm{c}} \otimes_{\mathrm{c}} M_{\mathrm{c}}$.

Now define $\varphi_{\mathrm{c}}$ and $\psi_{\mathrm{c}}$ to be the restriction of $\varphi$ and $\psi$ to $M_{\mathrm{c}}^{+}$respectively, giving you two faithful lower semi-continuous weights on $M_{\mathrm{c}}$.

By proposition 5.38 of [8] we get that $(I \otimes J) W(I \otimes J)=W^{*}$, implying that $R\left(\left(\iota \otimes \omega_{v, w}\right)(W)\right)=\left(\iota \otimes \omega_{J w, J v}\right)(W)$ for all $v, w \in H$. It follows that $R\left(M_{\mathrm{c}}\right)=M_{\mathrm{c}}$. Define $R_{\mathrm{c}}$ to be the restriction of $R$ to $M_{\mathrm{c}}$. Then $R_{\mathrm{c}}$ is a ${ }^{*}$-antiautomorphism of $M_{\mathrm{c}}$ satisfying $\chi\left(R_{\mathrm{c}} \otimes_{\mathrm{c}} R_{\mathrm{c}}\right) \Delta_{\mathrm{c}}=\Delta_{\mathrm{c}} R_{\mathrm{c}}$. It is also clear that $\psi_{\mathrm{c}}=\varphi_{\mathrm{c}} R_{\mathrm{c}}$.

For $a, b \in \mathscr{N}_{\psi}$ and $c \in \mathscr{N}_{\varphi}$, we have that

$$
\begin{aligned}
(\psi \otimes \iota)\left(\Delta\left(b^{*} c\right)(a \otimes 1)\right) & =R\left((\iota \otimes \varphi)\left((1 \otimes R(a)) \Delta\left(R(c) R(b)^{*}\right)\right)\right) \\
& =R\left(\left(\iota \otimes \omega_{\Lambda\left(R(c) R(b)^{*}\right), \Lambda\left(R(a)^{*}\right)}\right)\left(W^{*}\right)\right),
\end{aligned}
$$

which implies that $M_{\mathrm{c}}=\left[(\psi \otimes \iota)\left(\Delta\left(b^{*} c\right)(a \otimes 1)\right) \mid a, b \in \mathscr{N}_{\psi}, c \in \mathscr{N}_{\varphi}\right]$.

We know that $(\psi \otimes \iota)\left(\Delta\left(b^{*} c\right)(a \otimes 1)\right) \in \mathscr{N}_{\varphi}$ and thus $(\psi \otimes \iota)\left(\Delta\left(b^{*} c\right)(a \otimes\right.$ 1)) $\in \mathscr{N}_{\varphi_{\mathrm{c}}}$ for all $a, b \in \mathscr{N}_{\psi}$ and $c \in \mathscr{N}_{\varphi}$. It follows that $\varphi_{\mathrm{c}}$ is densely defined.

Define $\Lambda_{\mathrm{c}}$ to be the restriction of $\Lambda$ to $\mathscr{N}_{\varphi_{\mathrm{c}}}$. Equation (2) guarantees that $\Lambda_{\mathrm{c}}\left(\mathscr{N}_{\varphi_{\mathrm{c}}}\right)$ is dense in $H$. Therefore $\left(H, \iota, \Lambda_{\mathrm{c}}\right)$ is a GNS-construction for $\varphi_{\mathrm{c}}$. that

Proposition 5.38 of [8] tells us that $\left(N^{-1} \otimes \nabla\right) W=W\left(N^{-1} \otimes \nabla\right)$ implying

$$
\tau_{t}\left(\left(\iota \otimes \omega_{v, w}\right)(W)\right)=\left(\iota \otimes \omega_{\nabla^{i t} v, \nabla^{i t} w}\right)(W)
$$

for all $v, w \in H$ and $t \in \mathrm{R}$. Hence $\tau_{t}\left(M_{\mathrm{c}}\right)=M_{\mathrm{c}}$ for all $t \in \mathrm{R}$. Define the one-parameter group $\tau^{\mathrm{c}}$ on $M_{\mathrm{c}}$ by setting $\tau_{t}^{\mathrm{c}}=\tau_{t} \uparrow_{M_{\mathrm{c}}}$ for all $t \in \mathrm{R}$. Notice that equation (6) implies that $\tau^{\mathrm{c}}$ is norm continuous.

Since $\Delta_{\mathrm{c}}\left(M_{\mathrm{c}}\right)\left(1 \otimes M_{\mathrm{c}}\right)$ is a dense subset of $M_{\mathrm{c}} \otimes_{\mathrm{c}} M_{\mathrm{c}}$, we get that $M_{\mathrm{c}}=$ $\left[(\iota \otimes \omega) \Delta(x) \mid \omega \in B(H)_{*}, x \in M_{\mathrm{c}}\right.$ ]. Proposition 6.8 of [8] implies for all $t \in \mathrm{R}, \omega \in M_{*}$ and $x \in M_{\mathrm{c}}$ that

$$
\sigma_{t}((\iota \otimes \omega) \Delta(x))=\left(\iota \otimes \omega \sigma_{t}^{\prime}\right) \Delta\left(\tau_{t}^{\mathrm{c}}(x)\right) .
$$

Therefore $\sigma_{t}\left(M_{\mathrm{c}}\right)=M_{\mathrm{c}}$ for all $t \in \mathrm{R}$ and we can define a one parameter group $\sigma^{\mathrm{c}}$ on $M_{\mathrm{c}}$ by setting $\sigma_{t}^{\mathrm{c}}=\sigma_{t} \uparrow_{M_{\mathrm{c}}}$ for all $t \in \mathrm{R}$. Equation (7) implies that $\sigma^{\mathrm{c}}$ is norm continuous.

By now it is clear that $\varphi_{\mathrm{c}}$ is a KMS weight on $M_{\mathrm{c}}$ (in the $\mathrm{C}^{*}$-algebraic sense) with $\sigma^{\mathrm{c}}$ as its modular group. Because $\psi_{c}=\varphi_{c} R_{c}$, we also get that $\psi_{\mathrm{c}}$ is a KMS weight on $M_{\mathrm{c}}$.

Take $\omega \in\left(M_{\mathrm{c}}\right)_{+}^{*}$ and $x \in \mathscr{M}_{\varphi_{\mathrm{c}}}^{+}$. Choose $\eta \in B(H)_{*}^{+}$. On the $\mathrm{C}^{*}$-algebra $M_{\mathrm{c}}$ we can make a GNS-construction for the positive functional $\omega$. This way we obtain a Hilbert space $K$, a non-degenerate representation $\pi$ of $M_{\mathrm{c}}$ on $K$ and 
a (cyclic) vector $v \in K$ such that $\omega=\omega_{v, v} \pi$. By theorem 1.5 of [18] we know that $W$ belongs to the multiplier algebra of $M_{\mathrm{c}} \otimes B_{0}(H)$, where $B_{0}(H)$ denotes the $\mathrm{C}^{*}$-algebra of compact operators on $H$. Hence the unitary $U$ defined by $U:=\left(\pi \otimes_{\mathrm{c}} \iota\right)(W)$ belongs to $B(K) \otimes B(H)$. Define $\theta \in B(H)_{*}^{+}$by setting $\theta(x)=\left(\omega_{v, v} \otimes \eta\right)\left(U^{*}(1 \otimes x) U\right)$ for all $x \in B(H)$. Then

$$
\begin{aligned}
(\eta \otimes \iota) \Delta\left(\left(\omega \otimes_{\mathrm{c}} \iota\right)\left(\Delta_{\mathrm{c}}(x)\right)\right) & =(\eta \otimes \iota)\left(\left(\omega \otimes_{\mathrm{c}} \iota \otimes_{\mathrm{c}} \iota\right)\left(\left(\Delta_{\mathrm{c}} \otimes_{\mathrm{c}} \iota\right) \Delta_{\mathrm{c}}(x)\right)\right) \\
& =(\eta \otimes \iota)\left(\left(\omega_{v, v} \otimes \iota \otimes \iota\right)\left(U_{12}^{*} \Delta(x)_{23} U_{12}\right)\right) \\
& =(\theta \otimes \iota) \Delta(x) .
\end{aligned}
$$

Therefore the left invariance of $\varphi$ implies that $(\eta \otimes \iota) \Delta\left(\left(\omega \otimes_{c} \iota\right)\left(\Delta_{c}(x)\right)\right)$ belongs to $\mathscr{M}_{\varphi}^{+}$and

$$
\varphi\left((\eta \otimes \iota) \Delta\left(\left(\omega \otimes_{\mathrm{c}} \iota\right)\left(\Delta_{\mathrm{c}}(x)\right)\right)\right)=\theta(1) \varphi(x)=\omega(1) \eta(1) \varphi_{\mathrm{c}}(x) .
$$

Translating proposition 5.15 of [8] to the von Neumann algebra setting, we now conclude that $\left(\omega \otimes_{\mathrm{c}} \iota\right)\left(\Delta_{\mathrm{c}}(x)\right)$ belongs to $\mathscr{M}_{\varphi}^{+}$and therefore to $\mathscr{M}_{\varphi_{\mathrm{c}}}^{+}$.

Taking $\eta \in B(H)_{*}$ such that $\eta(1)=1$, equation (8) and the left invariance of $\varphi$ imply that

$$
\begin{aligned}
\varphi_{\mathrm{c}}\left(\left(\omega \otimes_{\mathrm{c}} \iota\right)\left(\Delta_{\mathrm{c}}(x)\right)\right) & =\varphi\left(\left(\omega \otimes_{\mathrm{c}} \iota\right)\left(\Delta_{\mathrm{c}}(x)\right)\right) \\
& =\varphi\left((\eta \otimes \iota) \Delta\left(\left(\omega \otimes_{\mathrm{c}} \iota\right)\left(\Delta_{\mathrm{c}}(x)\right)\right)\right) \\
& =\omega(1) \varphi_{\mathrm{c}}(x) .
\end{aligned}
$$

So we have proven that $\varphi_{\mathrm{c}}$ is left invariant in the sense of definition 2.2 of [8]. Because $\chi\left(R_{\mathrm{c}} \otimes_{\mathrm{c}} R_{\mathrm{c}}\right) \Delta=\Delta R_{\mathrm{c}}$ and $\psi_{\mathrm{c}}=\varphi_{\mathrm{c}} R_{\mathrm{c}}$ we also get that $\psi_{\mathrm{c}}$ is right invariant. From all this we conclude that $\left(M_{\mathrm{c}}, \Delta_{\mathrm{c}}\right)$ is a reduced $\mathrm{C}^{*}$-algebraic quantum group.

The GNS-construction $\left(H, \iota, \Lambda_{\mathrm{c}}\right)$ for $\varphi_{\mathrm{c}}$ was obtained by letting $\Lambda_{\mathrm{c}}$ be the restriction of $\Lambda$ to $\mathscr{N}_{\varphi_{\mathrm{c}}}$. By the definitions introduced at the end of section 4 in [8], it is clear that this implies that $W$ is the multiplicative unitary of $\left(M_{\mathrm{c}}, \Delta_{\mathrm{c}}\right)$ in this GNS-construction $\left(H, \iota, \Lambda_{\mathrm{c}}\right)$.

Since $\sigma_{t}^{\mathrm{c}}$ and $\tau_{t}^{\mathrm{c}}$ are restrictions of $\sigma_{t}$ and $\tau_{t}$ respectively, it is clear that $\left(\tau_{t}^{\mathrm{c}} \otimes \sigma_{t}^{\mathrm{c}}\right) \Delta=\Delta \sigma_{t}^{\mathrm{c}}$ for all $t \in \mathrm{R}$, and so the density conditions imply that $\tau^{\mathrm{c}}$ is the scaling group of $\left(M_{\mathrm{c}}, \Delta_{\mathrm{c}}\right)$. It also follows that $v$ is the scaling constant of $\left(M_{\mathrm{c}}, \Delta_{\mathrm{c}}\right)$. Letting $S_{\mathrm{c}}$ denote the antipode of $\left(M_{\mathrm{c}}, \Delta_{\mathrm{c}}\right)$, proposition 5.33 of [8] and its von Neumann algebraic counterpart imply that $S_{\mathrm{c}} \subseteq S$. Since $\tau^{\mathrm{c}}$ is the scaling group of $\left(M_{\mathrm{c}}, \Delta_{\mathrm{c}}\right)$ and $R_{\mathrm{c}}$ was obtained by restricting $R$ to $M_{\mathrm{c}}$, this implies that $R_{\mathrm{c}}$ is the unitary antipode of $\left(M_{\mathrm{c}}, \Delta_{\mathrm{c}}\right)$. 
Following [8], we associate to the reduced $\mathrm{C}^{*}$-algebraic quantum group $\left(M_{\mathrm{c}}, \Delta_{\mathrm{c}}\right)$ the von Neumman algebraic quantum group $\left(\tilde{M}_{\mathrm{c}}, \tilde{\Delta}_{\mathrm{c}}\right)$ by letting $\tilde{M}_{\mathrm{c}}$ be the $\sigma$-strong* closure of $M_{\mathrm{c}}$ and defining $\tilde{\Delta}_{\mathrm{c}}$ to be the unique normal ${ }^{*}$-homomorphism from $\tilde{M}_{\mathrm{c}}$ to $\tilde{M}_{\mathrm{c}} \otimes \tilde{M}_{\mathrm{c}}$ extending $\Delta_{\mathrm{c}}$. It follows from proposition 1.4 that $\left(\tilde{M}_{\mathrm{c}}, \tilde{\Delta}_{\mathrm{c}}\right)=(M, \Delta)$. We get similar results for the extensions of the Haar weights, their modular groups, the scaling group, the unitary antipode and the antipode itself.

\section{Commutation relations and related matters}

In this section we establish some useful technical properties about von Neumann algebraic quantum groups that are often used when working in the operator algebra approach to quantum groups. We start off by implementing the scaling groups and unitary antipodes. Then we prove some results concerning the dual and end by formulating some commutation relations.

Proposition 2.1. The following properties hold for $t \in \mathbf{R}$

$$
\begin{aligned}
& \tau_{t}(x)=\hat{\nabla}^{i t} x \hat{\nabla}^{-i t}=P^{i t} x P^{-i t} \quad \text { and } \quad R(x)=\hat{J} x^{*} \hat{J} \quad \text { for all } \quad x \in M \\
& \hat{\tau}_{t}(x)=\nabla^{i t} x \nabla^{-i t}=P^{i t} x P^{-i t} \quad \text { and } \quad \hat{R}(x)=J x^{*} J \quad \text { for all } \quad x \in \hat{M}
\end{aligned}
$$

Proof. By propositions 8.17 and 8.25 of [8], we know that $\hat{R}(x)=J x^{*} J$ for all $x \in \hat{M}$. Therefore the Pontryagin duality theorem guarantees that also $R(x)=\hat{J} x^{*} \hat{J}$ for all $x \in M$.

Choose $x \in M$. By lemma 8.8 and proposition 8.9 of [8] we know that $\hat{\nabla}^{i t}=P^{i t} J \delta^{i t} J$, and so we get that $\hat{\nabla}^{i t} x \hat{\nabla}^{-i t}=P^{i t} J \delta^{i t} J x J \delta^{-i t} J P^{-i t}$. But Tomita-Takesaki theory tells us that $J \delta^{i t} J$ belongs to $M^{\prime}$, implying that $\hat{\nabla}^{i t} x \hat{\nabla}^{-i t}=P^{i t} x P^{-i t}=\tau_{t}(x)$.

Pontryagin duality allows us to conclude that $\hat{\tau}_{t}(x)=\nabla^{i t} x \nabla^{-i t}$ for all $t \in \mathrm{R}$ and $x \in \hat{M}$.

We have that $(R \otimes \hat{R})(W)=W$ and $\left(\tau_{t} \otimes \hat{\tau}_{t}\right)(W)=W$ for all $t \in \mathbf{R}$ (see the remarks before propositions 8.18 and 8.25 of [8]). Hence the next result.

COROLlary 2.2. We have the following commutation relations:

$$
W(\hat{\nabla} \otimes \nabla)=(\hat{\nabla} \otimes \nabla) W \quad \text { and } \quad W(\hat{J} \otimes J)=(\hat{J} \otimes J) W^{*} .
$$

Notice that for the same reasons, $W(P \otimes \nabla)=(P \otimes \nabla) W$ and $W(\hat{\nabla} \otimes P)=$ $(\hat{\nabla} \otimes P) W$.

In the next part, we complete the picture of the dual. First we introduce a natural ${ }^{*}$-algebra inside $M_{*}$. 
Definition 2.3. Define the subspace $M_{*}^{\sharp}$ of $M_{*}$ as

$$
M_{*}^{\sharp}=\left\{\omega \in M_{*} \mid \exists \theta \in M_{*}: \theta(x)=\bar{\omega}(S(x)) \text { for all } x \in D(S)\right\} .
$$

We define the antilinear mapping $.^{*}: M_{*}^{\sharp} \rightarrow M_{*}^{\sharp}$ such that $\omega^{*}(x)=\bar{\omega}(S(x))$ for all $\omega \in M_{*}^{\sharp}$ and $x \in D(S)$. Then $M_{*}^{\sharp}$ is a subalgebra of $M_{*}$ and becomes a *-algebra under the operation.$^{*}$.

If $x \in D(S)$, then $S(x)^{*} \in D(S)$ and $S\left(S(x)^{*}\right)^{*}=x$ (which follows from the corresponding property for $\tau_{-\frac{i}{2}}$ ). Using lemma 5.25 of [8], one shows that $M_{*}^{\sharp}$ is a subalgebra of $M_{*}$ and that .* is antimultiplicative. Notice that, since $S$ can be unbounded, $M_{*}^{\sharp}$ can be strictly smaller than $M_{*}$.

The space $\left\{(\iota \otimes \omega)(W) \mid \omega \in B(H)_{*}\right\}$ is a $\sigma$-strong* core for $S$ and $S((\iota \otimes \omega)(W))=(\iota \otimes \omega)\left(W^{*}\right)$ for $\omega \in B(H)_{*}$. (see proposition 8.3 of [8]). We use this characterization of $S$ to prove the next result.

Proposition 2.4. The following holds :

1. $M_{*}^{\sharp}=\left\{\omega \in M_{*} \mid \exists \theta \in M_{*}: \lambda(\omega)^{*}=\lambda(\theta)\right\}$.

2. $\lambda(\omega)^{*}=\lambda\left(\omega^{*}\right)$ for all $\omega \in M_{*}^{\sharp}$.

Proof. Take $\omega \in M_{*}$. Then we have for all $\eta \in B(H)_{*}$ that

$$
\bar{\omega}(S((\iota \otimes \eta)(W)))=\bar{\omega}\left((\iota \otimes \eta)\left(W^{*}\right)\right)=\eta\left((\omega \otimes \iota)(W)^{*}\right)=\eta\left(\lambda(\omega)^{*}\right)
$$

If $\omega \in M_{*}^{\sharp}$ then the formula above implies for all $\eta \in B(H)_{*}$ that

$$
\eta\left(\lambda\left(\omega^{*}\right)\right)=\eta\left(\left(\omega^{*} \otimes \iota\right)(W)\right)=\omega^{*}((\iota \otimes \eta)(W))=\eta\left(\lambda(\omega)^{*}\right)
$$

and hence $\lambda\left(\omega^{*}\right)=\lambda(\omega)^{*}$.

Now suppose that there exists $\theta \in M_{*}$ such that $\lambda(\omega)^{*}=\lambda(\theta)$. By formula (9) above, we get for all $\eta \in B(H)_{*}$ that

$$
\bar{\omega}(S((\iota \otimes \eta)(W)))=\eta((\theta \otimes \iota)(W))=\theta((\iota \otimes \eta)(W)) .
$$

Because such elements $(\iota \otimes \eta)(W)$ form a $\sigma$-strong* core for $S$, we get $\bar{\omega}(S(x))=\theta(x)$ for all $x \in D(S)$. So $\omega$ belongs to $M_{*}^{\sharp}$.

It is easy to prove that $M_{*}^{\sharp}$ is dense in $M_{*}$ implying that the ${ }^{*}$-algebra $\lambda\left(M_{*}^{\sharp}\right)$ is $\sigma$-strong* dense in $\hat{M}$. For later purposes, we will need a result which gives a little bit more information.

Lemma 2.5. The spaces $\mathscr{I} \cap M_{*}^{\sharp}$ and $\left(\mathscr{I} \cap M_{*}^{\sharp}\right)^{*}$ are dense in $M_{*}$ and $\lambda\left(\mathscr{I} \cap M_{*}^{\sharp}\right)$ is a $\sigma$-strong ${ }^{*}$-norm core for $\hat{\Lambda}$. 
Proof. Consider $\omega \in \mathscr{I}$. For every $n \in \mathbf{N}$ and $z \in \mathbf{C}$, we define $\omega(n, z) \in$ $M_{*}$ as

$$
\omega(n, z)=\frac{n}{\sqrt{\pi}} \int \exp \left(-n^{2}(t+z)^{2}\right) \omega \tau_{t} d t .
$$

So we have for $x \in D(S)$ that $x \in D\left(\tau_{-\frac{i}{2}}\right)$ and thus

$$
\begin{aligned}
\overline{\omega(n, z)}(S(x)) & =\frac{n}{\sqrt{\pi}} \int \exp \left(-n^{2}(t+\bar{z})^{2}\right) \bar{\omega}\left(\tau_{t}(S(x))\right) d t \\
& =\frac{n}{\sqrt{\pi}} \int \exp \left(-n^{2}(t+\bar{z})^{2}\right) \bar{\omega}\left(R\left(\tau_{t-\frac{i}{2}}(x)\right)\right) d t \\
& =\frac{n}{\sqrt{\pi}} \int \exp \left(-n^{2}\left(t+\frac{i}{2}+\bar{z}\right)^{2}\right) \bar{\omega}\left(R\left(\tau_{t}(x)\right)\right) d t
\end{aligned}
$$

from which we conclude that $\omega(n, z) \in M_{*}^{\sharp}$ and

$$
\omega(n, z)^{*}=\frac{n}{\sqrt{\pi}} \int \exp \left(-n^{2}\left(t+\frac{i}{2}+\bar{z}\right)^{2}\right) \bar{\omega} R \tau_{t} d t .
$$

It is easy to check that for every $t \in \mathrm{R}$ we have $\omega \tau_{t} \in \mathscr{I}$ and $\xi\left(\omega \tau_{t}\right)=$ $v^{-\frac{t}{2}} P^{-i t} \xi(\omega)$. Therefore the closedness of the mapping $\eta \mapsto \xi(\eta)$ implies that $\omega(n, z) \in \mathscr{I}$ and

$$
\xi(\omega(n, z))=\frac{n}{\sqrt{\pi}} \int \exp \left(-n^{2}(t+z)^{2}\right) v^{-\frac{t}{2}} P^{-i t} \xi(\omega) d t
$$

(1) Let $\omega \in \mathscr{I}$. Then we have for every $n \in \mathrm{N}$ that $\omega(n, 0) \in \mathscr{I} \cap M_{*}^{\sharp}$. Clearly, $(\omega(n, 0))_{n=1}^{\infty}$ converges to $\omega$. Equation $(11)$ implies that $(\xi(\omega(n, 0)))_{n=1}^{\infty}$ converges to $\xi(\omega)$. In other words, $(\hat{\Lambda}(\lambda(\omega(n, 0))))_{n=1}^{\infty}$ converges to $\hat{\Lambda}(\lambda(\omega))$. Since $\mathscr{I}$ is dense in $M_{*}$ and $\lambda(\mathscr{I})$ is a core for $\hat{\Lambda}$, we conclude that $\mathscr{I} \cap M_{*}^{\sharp}$ is dense in $M_{*}$ and that $\lambda\left(\mathscr{I} \cap M_{*}^{\sharp}\right)$ is a $\sigma$-strong*-norm core for $\hat{\Lambda}$.

(2) Let $\omega \in \mathscr{I}$. Then we have for every $n \in \mathrm{N}$ that $\omega\left(n, \frac{i}{2}\right) \in \mathscr{I} \cap M_{*}^{\sharp}$ and

$$
\omega\left(n, \frac{i}{2}\right)^{*}=\frac{n}{\sqrt{\pi}} \int \exp \left(-n^{2} t^{2}\right) \bar{\omega} R \tau_{t} d t
$$

by equation (10). So $\left(\omega\left(n, \frac{i}{2}\right)^{*}\right)_{n=1}^{\infty}$ converges to $\bar{\omega} R$. From this all, we conclude that $\left(\mathscr{I} \cap M_{*}^{\sharp}\right) *$ is dense in $M_{*}$.

Proposition 2.6. Define $\mathscr{I}^{\sharp}=\left\{\omega \in \mathscr{I} \cap M_{*}^{\sharp} \mid \omega^{*} \in \mathscr{I}\right\}$. Then $\mathscr{I}^{\sharp}$ is $a^{*}$ subalgebra of $M_{*}^{\sharp}$ such that $\mathscr{I}^{\sharp}$ is dense in $M_{*}$ and $\lambda\left(\mathscr{I}^{\sharp}\right)$ is a $\sigma$-strong ${ }^{*}$-norm core for $\hat{\Lambda}$. 
Proof. It is clear that $\mathscr{I}^{\sharp}$ is a ${ }^{*}$-subalgebra of $M_{*}^{\sharp}$. Because $\mathscr{I}$ is a left ideal in $M_{*}$, we get that $\left(\mathscr{I} \cap M_{*}^{\sharp}\right)^{*}\left(\mathscr{I} \cap M_{*}^{\sharp}\right) \subseteq \mathscr{I}^{\sharp}$. Thus in order to prove that $\mathscr{I}^{\sharp}$ is dense in $M_{*}$, it is by the previous lemma enough to prove that $\left(M_{*}\right)^{2}$ is dense in $M_{*}$. But we have for all $v \in H$ with $\|v\|=1, w_{1}, w_{2} \in H$ and $x \in M$ that

$$
\left\langle\Delta(x) W^{*}\left(v \otimes w_{1}\right), W^{*}\left(v \otimes w_{2}\right)\right\rangle=\left\langle x w_{1}, w_{2}\right\rangle,
$$

which easily implies that $\left(M_{*}\right)^{2}$ is dense in $M_{*}$. Hence $\mathscr{I}^{\sharp}$ is dense in $M_{*}$.

Since $\mathscr{I} \cap M_{*}^{\sharp}$ is dense in $M_{*}, 1$ belongs to the $\sigma$-strong* closure of $\lambda(I \cap$ $\left.M_{*}^{\sharp}\right)^{*}$. Combining this with the fact that $\lambda\left(I \cap M_{*}^{\sharp}\right)$ is a $\sigma$-strong*-norm core for $\hat{\Lambda}$ and the inclusion $\lambda\left(\mathscr{I} \cap M_{*}^{\sharp}\right)^{*} \lambda\left(\mathscr{I} \cap M_{*}^{\sharp}\right) \subseteq \lambda\left(\mathscr{I}^{\sharp}\right)$, we conclude that $\lambda\left(\mathscr{I}^{\sharp}\right)$ is a $\sigma$-strong ${ }^{*}$-norm core for $\hat{\Lambda}$.

Let us connect the modular objects of $\hat{\varphi}$ to objects already constructed on the level of $(M, \Delta)$.

We know that the operators $P$ and $J \delta J$ strongly commute and that $\hat{\nabla}^{i t}=$ $P^{i t} J \delta^{i t} J$ for $t \in \mathrm{R}$ (see lemma 8.8 and proposition 8.9 of [8]). Also notice that this implies for every $a \in \mathscr{N}_{\varphi}$ that $\tau_{t}(a) \delta^{-i t}$ belongs to $\mathscr{N}_{\varphi}$ and $\hat{\nabla}^{i t} \Lambda(a)=$ $\Lambda\left(\tau_{t}(a) \delta^{-i t}\right)$.

Put $\hat{T}=\hat{J} \hat{\nabla}^{\frac{1}{2}}$. So $\hat{\Lambda}\left(\mathscr{N}_{\hat{\varphi}} \cap \mathscr{N}_{\hat{\varphi}}^{*}\right)$ is a core for $\hat{T}$ and $\hat{T} \hat{\Lambda}(x)=\hat{\Lambda}\left(x^{*}\right)$ for all $x \in \mathscr{N}_{\hat{\varphi}} \cap \mathscr{N}_{\hat{\varphi}}^{*}$.

\section{Lemma 2.7. The set $\hat{\Lambda}\left(\lambda\left(\mathscr{I}^{\sharp}\right)\right)$ is a core for $\hat{T}$.}

Proof. Since $\hat{T}=\hat{J} \hat{\nabla}^{\frac{1}{2}}$, the definition of $\hat{T}$ gives clearly that $\hat{\Lambda}\left(\lambda\left(\mathscr{I}^{\sharp}\right)\right) \subseteq$ $D\left(\hat{\nabla}^{\frac{1}{2}}\right)$. From proposition 2.6, we know that $\hat{\Lambda}\left(\lambda\left(\mathscr{I}^{\sharp}\right)\right)$ is a dense subspace in $H$.

We now use the notation $\rho_{t}$ as it was introduced in notation 8.7 of [8]. For every $\omega \in M_{*}$ we denote by $\rho_{t}(\omega)$ the element in $M_{*}$ defined by $\rho_{t}(\omega)(x)=$ $\omega\left(\delta^{-i t} \tau_{-t}(x)\right)$. Then $\rho_{t}(\mathscr{I})=\mathscr{I}$ and $\xi\left(\rho_{t}(\omega)\right)=\hat{\nabla}^{i t} \xi(\omega)$ for all $\omega \in \mathscr{I}$ and $t \in \mathrm{R}$. If $\omega \in M_{*}^{\sharp}$ and $t \in \mathrm{R}$, it is not so difficult to check that $\rho_{t}(\omega) \in M_{*}^{\sharp}$ and $\rho_{t}(\omega)^{*}=\rho_{t}\left(\omega^{*}\right)$. It follows that $\rho_{t}\left(\mathscr{I}^{\sharp}\right)=\mathscr{I}^{\sharp}$ for all $t \in \mathbf{R}$, hence $\hat{\sigma}_{t}\left(\lambda\left(\mathscr{I}^{\sharp}\right)\right)=\lambda\left(\rho_{t}\left(\mathscr{I}^{\sharp}\right)\right)=\lambda\left(\mathscr{I}^{\sharp}\right)$ for all $t \in \mathrm{R}$.

Therefore $\hat{\nabla}^{i t} \hat{\Lambda}\left(\lambda\left(\mathscr{I}^{\sharp}\right)\right)=\hat{\Lambda}\left(\lambda\left(\mathscr{I}^{\sharp}\right)\right)$ for all $t \in \mathrm{R}$. We conclude from all this that $\hat{\Lambda}\left(\lambda\left(\mathscr{I}^{\sharp}\right)\right)$ is a core for $\hat{\nabla}^{\frac{1}{2}}$ (see e.g. corollary 1.21 of [7]) and the lemma follows.

Proposition 2.8. Consider $x \in \mathscr{N}_{\varphi} \cap D\left(S^{-1}\right)$ such that $S^{-1}(x)^{*} \in \mathscr{N}_{\varphi}$. Then $\Lambda(x) \in D\left(\hat{T}^{*}\right)$ and $\hat{T}^{*} \Lambda(x)=\Lambda\left(S^{-1}(x)^{*}\right)$. 
Proof. Choose $\theta \in \mathscr{I}^{\sharp}$. Then

$$
\begin{aligned}
\langle\hat{T} \hat{\Lambda}(\lambda(\theta)), \Lambda(x)\rangle & =\left\langle\hat{\Lambda}\left(\lambda(\theta)^{*}\right), \Lambda(x)\right\rangle \\
& =\left\langle\hat{\Lambda}\left(\lambda\left(\theta^{*}\right)\right), \Lambda(x)\right\rangle \\
& =\left\langle\xi\left(\theta^{*}\right), \Lambda(x)\right\rangle .
\end{aligned}
$$

Therefore the definition of $\xi\left(\theta^{*}\right)$ and $\theta^{*}$ imply that

$$
\begin{aligned}
\langle\hat{T} \hat{\Lambda}(\lambda(\theta)), \Lambda(x)\rangle & =\theta^{*}\left(x^{*}\right)=\bar{\theta}\left(S\left(x^{*}\right)\right)=\overline{\theta\left(S^{-1}(x)\right)} \\
& =\overline{\left\langle\xi(\theta), \Lambda\left(S^{-1}(x)^{*}\right)\right\rangle}=\left\langle\Lambda\left(S^{-1}(x)^{*}\right), \xi(\theta)\right\rangle \\
& =\left\langle\Lambda\left(S^{-1}(x)^{*}\right), \hat{\Lambda}(\lambda(\theta))\right\rangle .
\end{aligned}
$$

Thus the previous lemma implies that $\Lambda(x)$ belongs to $D\left(\hat{T}^{*}\right)$ and $\hat{T}^{*} \Lambda(x)=$ $\Lambda\left(S^{-1}(x)^{*}\right)$.

This proposition allows us to establish easily a connection between $G$ and $\hat{T}$. Recall that the operators $G, N$ and $I$ were introduced in proposition 1.3.

Corollary 2.9. We have that $\hat{T}^{*}=G, \hat{\nabla}=N^{-1}$ and $\hat{J}=I$.

Proof. Using proposition 1.3 and the strong left invariance of $\psi$ (see proposition 5.24 of [8]), the previous result implies easily $G \subseteq \hat{T}^{*}$.

Define the subspace $C$ of $D(G)$ as $C=\left\langle\Lambda\left((\psi \otimes \iota)\left(\Delta\left(y^{*}\right)(x \otimes 1)\right)\right)\right|$ $\left.x, y \in \mathscr{N}_{\varphi}^{*} \mathscr{N}_{\psi}\right\rangle$.

Let $t \in \mathrm{R}$. Remember that $\hat{\nabla}^{i t} \Lambda(a)=\Lambda\left(\tau_{t}(a) \delta^{-i t}\right)$ for all $a \in \mathscr{N}_{\varphi}$.

Choose $x, y \in \mathscr{N}_{\varphi}^{*} \mathscr{N}_{\psi}$. Then $\delta^{i t} \tau_{t}(x)$ and $\delta^{i t} \tau_{t}(y)$ belong to $\mathscr{N}_{\varphi}^{*} \mathscr{N}_{\psi}$ and $\tau_{t}\left((\psi \otimes \iota)\left(\Delta\left(y^{*}\right)(x \otimes 1)\right)\right) \delta^{-i t}=v^{t}(\psi \otimes \iota)\left(\Delta\left(\left(\delta^{i t} \tau_{t}(y)\right)^{*}\right)\left(\delta^{i t} \tau_{t}(x) \otimes 1\right)\right)$.

Therefore the element

$$
\hat{\nabla}^{i t} \Lambda\left((\psi \otimes \iota)\left(\Delta\left(y^{*}\right)(x \otimes 1)\right)\right)=\Lambda\left(\tau_{t}\left((\psi \otimes \iota)\left(\Delta\left(y^{*}\right)(x \otimes 1)\right)\right) \delta^{-i t}\right)
$$

belongs to $C$.

We conclude that $C$ is a dense subspace of $D\left(\hat{\nabla}^{-\frac{1}{2}}\right)$, invariant under the family of operators $\hat{\nabla}^{i t}(t \in \mathrm{R})$. It follows that $C$ is a core for $\hat{\nabla}^{-\frac{1}{2}}$ and thus a core for $\hat{T}^{*}=\hat{J} \hat{\nabla}^{-\frac{1}{2}}$. Combining this with the fact that $G \subseteq \hat{T}^{*}$, we conclude that $G=\hat{T}^{*}$. Now the uniqueness of the polar decomposition implies that $\hat{\nabla}=N^{-1}$ and $\hat{J}=I$.

Combining the previous corollary with proposition 1.3 we get the following. 
Corollary 2.10. The set

$$
\left\{\Lambda(x) \mid x \in \mathscr{N}_{\varphi} \cap D\left(S^{-1}\right) \text { such that } S^{-1}(x)^{*} \in \mathscr{N}_{\varphi}\right\}
$$

is a core for $\hat{T}^{*}$.

Recall that we introduced the GNS-construcion $(H, \iota, \Gamma)$ for $\psi$ by considering $\psi$ as $\varphi_{\delta}$ and setting $\Gamma=\Lambda_{\delta}$. But $\psi$ is by definition equal to $\varphi R$. It turns out that $\hat{J}$ connects both pictures of $\psi$ :

Proposition 2.11. We have for all $x \in \mathscr{N}_{\psi}$ that $\hat{J} \Gamma(x)=\Lambda\left(R(x)^{*}\right)$.

Proof. Define the anti-unitary $U: H \rightarrow H$ such that $U \Gamma(x)=\Lambda\left(R(x)^{*}\right)$ for $x \in \mathscr{N}_{\psi}$. Choose $a \in \mathscr{N}_{\varphi}$ such that $a \in D\left(S^{-1}\right)$ and $S^{-1}(a)^{*} \in \mathscr{N}_{\varphi}$.

For $n \in \mathrm{N}$, we define $e_{n} \in M$ such that $e_{n}=\frac{n}{\sqrt{\pi}} \int \exp \left(-n^{2} t^{2}\right) \delta^{i t} d t$. Remember that $e_{n}$ is analytic with respect to $\sigma$ and $\sigma^{\prime}$, implying that $\mathscr{N}_{\varphi} e_{n} \subseteq$ $\mathscr{N}_{\varphi}$ and $\mathscr{N}_{\psi} e_{n} \subseteq \mathscr{N}_{\psi}$

Since $\tau_{s}(\delta)=\delta$ we see that $\tau_{s}\left(e_{n}\right)=e_{n}$ for $s \in \mathrm{R}$, hence $e_{n} \in D\left(\tau_{\frac{i}{2}}\right)$ and $\tau_{\frac{i}{2}}\left(e_{n}\right)=e_{n}$. By assumption, $a \in D\left(\tau_{\frac{i}{2}}\right)$, so $a e_{n} \in D\left(\tau_{\frac{i}{2}}\right)$ and $\tau_{\frac{i}{2}}\left(a e_{n}\right)=$ $\tau_{\frac{i}{2}}(a) e_{n}$. Hence $\tau_{\frac{i}{2}}\left(a e_{n}\right) \delta^{\frac{1}{2}}$ is a bounded operator and its closure equals $\tau_{\frac{i}{2}}(a)\left(\delta^{\frac{1}{2}} e_{n}\right)$.

Define the strongly continuous one-parameter group $\kappa$ of isometries of $M$ such that $\kappa_{t}(x)=\tau_{t}(x) \delta^{-i t}$ for $x \in M$ and $t \in \mathrm{R}$. The discussion above implies (see e.g. proposition 4.9 of [7]) that $a e_{n} \in D\left(\kappa_{\frac{i}{2}}\right)$ and $\kappa_{\frac{i}{2}}\left(a e_{n}\right)=\tau_{\frac{i}{2}}(a)\left(\delta^{\frac{1}{2}} e_{n}\right)$.

By assumption $R\left(\tau_{\frac{i}{2}}(a)\right)^{*}=S^{-1}(a)^{*} \in \mathscr{N}_{\varphi}$, implying that $\tau_{\frac{i}{2}}^{2}(a)$ belongs to $\mathscr{N}_{\psi}$. So we see that $\kappa_{\frac{i}{2}}\left(a e_{n}\right) \delta^{-\frac{1}{2}}$ is a bounded operator and that its closure equals $\tau_{\frac{i}{2}}(a) e_{n} \in \mathscr{N}_{\psi}$. Since $\Lambda=\Gamma_{\delta^{-1}}$, this implies that $\kappa_{\frac{i}{2}}\left(a e_{n}\right) \in \mathscr{N}_{\varphi}$ and

$$
\Lambda\left(\kappa_{\frac{i}{2}}\left(a e_{n}\right)\right)=\Gamma\left(\kappa_{\frac{i}{2}}\left(a e_{n}\right) \delta^{-\frac{1}{2}}\right)=\Gamma\left(\tau_{\frac{i}{2}}(a) e_{n}\right) .
$$

We know that we have for every $x \in \mathscr{N}_{\varphi}$ that $\kappa_{t}(x) \in \mathscr{N}_{\varphi}$ and $\Lambda\left(\kappa_{t}(x)\right)=$ $\hat{\nabla}^{i t} \Lambda(x)$. Since $a e_{n} \in \mathscr{N}_{\varphi}$ and $\kappa_{\frac{i}{2}}\left(a e_{n}\right) \in \mathscr{N}_{\varphi}$, we conclude (see e.g. proposition 4.4 of [6]) that $\Lambda\left(a e_{n}\right) \in D\left(\hat{\nabla}^{-\frac{1}{2}}\right)$ and

$$
\hat{\nabla}^{-\frac{1}{2}} \Lambda\left(a e_{n}\right)=\Lambda\left(\kappa_{\frac{i}{2}}\left(a e_{n}\right)\right)=\Gamma\left(\tau_{\frac{i}{2}}(a) e_{n}\right) .
$$

Since $\left(\Lambda\left(a e_{n}\right)\right)_{n=1}^{\infty}$ converges to $\Lambda(a)$ and $\left(\Gamma\left(\tau_{\frac{i}{2}}(a) e_{n}\right)\right)_{n=1}^{\infty}$ converges to $\Gamma\left(\tau_{\frac{i}{2}}(a)\right)$, the closedness of $\hat{\nabla}^{-\frac{1}{2}}$ implies that $\Lambda(a) \in D\left(\hat{\nabla}^{-\frac{1}{2}}\right)$ and

$$
\hat{\nabla}^{-\frac{1}{2}} \Lambda(a)=\Gamma\left(\tau_{\frac{i}{2}}(a)\right) .
$$


Consequently

$$
\begin{aligned}
U \hat{\nabla}^{-\frac{1}{2}} \Lambda(a)=U \Gamma\left(\tau_{\frac{i}{2}}(a)\right)= & \Lambda\left(R\left(\tau_{\frac{i}{2}}(a)\right)^{*}\right) \\
& =\Lambda\left(S^{-1}(a)^{*}\right)=\hat{T}^{*} \Lambda(a)=\hat{J} \hat{\nabla}^{-\frac{1}{2}} \Lambda(a) .
\end{aligned}
$$

Since such elements $\Lambda(a)$ form a core for $\hat{\nabla}^{-\frac{1}{2}}=\hat{J} \hat{T}^{*}$, such elements $\hat{\nabla}^{-\frac{1}{2}} \Lambda(a)$ form a dense subspace of $H$. Therefore $\hat{J}=U$ and we are done.

The equality in the next corollary is a slight adaptation of corollary 3.6.2.(iv) of [11].

COROLlary 2.12. We have that $\hat{J} J=v^{\frac{i}{4}} J \hat{J}$.

Proof. By [14], $J^{\prime}:=v^{\frac{i}{4}} J$ is the modular conjugation of $\psi$ in the GNSconstruction $(H, \iota, \Gamma)$. Choose $x \in \mathscr{N}_{\psi} \cap D\left(\sigma_{\frac{i}{2}}^{\prime}\right)$. Since $\sigma_{-t} R=R \sigma_{t}^{\prime}$ for all $t \in \mathrm{R}$, we get that $R(x) \in D\left(\sigma_{-\frac{i}{2}}\right)$ and $\sigma_{-\frac{i}{2}}(R(x))=R\left(\sigma_{\frac{i}{2}}^{\prime}(x)\right)$. Hence $R(x)^{*} \in \mathscr{N}_{\varphi} \cap D\left(\sigma_{\frac{i}{2}}\right)$ and $\sigma_{\frac{i}{2}}\left(R(x)^{*}\right)=R\left(\sigma_{\frac{i}{2}}^{\prime}(x)\right)^{*}$. Combining this with the previous proposition and the definition of the modular conjugation, we get that

$$
\begin{aligned}
\hat{J} J \Gamma(x) & =v^{\frac{i}{4}} \hat{J} J^{\prime} \Gamma(x)=v^{\frac{i}{4}} \hat{J} \Gamma\left(\sigma_{\frac{i}{2}}^{\prime}(x)^{*}\right)=v^{\frac{i}{4}} \Lambda\left(R\left(\sigma_{\frac{i}{2}}^{\prime}(x)^{*}\right)^{*}\right) \\
& =v^{\frac{i}{4}} \Lambda\left(\sigma_{\frac{i}{2}}\left(R(x)^{*}\right)^{*}\right)=v^{\frac{i}{4}} J \Lambda\left(R(x)^{*}\right)=v^{\frac{i}{4}} J \hat{J} \Gamma(x) .
\end{aligned}
$$

Therefore $\hat{J} J=v^{\frac{i}{4}} J \hat{J}$.

Let us denote the modular operator of $\psi$ in the GNS-construction $(H, \iota, \Gamma)$ by $\nabla$ and the modular operator of $\hat{\psi}$ in the GNS-construction $(H, \iota, \hat{\Gamma})$ by $\hat{\nabla}$.

Proposition 2.13. For all $s, t \in \mathrm{R}$ we have the following commutation relations.

$$
\begin{aligned}
& \hat{\nabla}^{i t} \nabla^{i s}=v^{i s t} \nabla^{i s} \hat{\nabla}^{i t} \text { and } \hat{\nabla}^{i t} \nabla^{i s}=v^{i s t} \nabla^{i s} \hat{\nabla}^{i t} \\
& \hat{\nabla}^{i t} \nabla^{i s}=v^{i s t} \nabla^{i s} \hat{\nabla}^{i t} \text { and } \nabla^{i s} \nabla^{i t}=\nabla^{i t} \nabla^{i s} \\
& \hat{J} \nabla \hat{J}=\nabla, \quad J \nabla J=\nabla^{-1} \text { and } J \nabla J=\nabla^{-1} \\
& \hat{J} P \hat{J}=P^{-1} \text { and } \hat{J} \delta \hat{J}=\delta^{-1} \\
& P^{i s} \nabla^{i t}=\nabla^{i t} P^{i s} \text { and } P^{i s} \nabla^{i t}=\nabla^{i t} P^{i s} \\
& P^{i s} \delta^{i t}=\delta^{i t} P^{i s} \quad \nabla^{i s} \delta^{i t}=v^{i s t} \delta^{i t} \nabla^{i s} \text { and } \nabla^{i s} \delta^{i t}=v^{i s t} \delta^{i t} \nabla^{i s} \\
& \hat{\nabla}^{i s} \delta^{i t}=\delta^{i t} \hat{\nabla}^{i s} \text { and } \hat{\nabla}^{i s} \delta^{i t}=\delta^{i t} \hat{\nabla}^{i s}
\end{aligned}
$$


All commutation relations remain true if we remove the ${ }^{\wedge}$ of $\nabla, \nabla$ and $J$ if there is one, add $a^{\wedge}$ to $\nabla, \nabla$ and $J$ if there is not one, replace $v$ by $v^{-1}$, replace $\delta$ by $\hat{\delta}$ and leave $P$ unchanged.

Proof. It is easy to check that $\nabla^{i t} \Lambda(x)=v^{-\frac{t}{2}} \Lambda\left(\sigma_{t}^{\prime}(x)\right)$ for all $x \in \mathscr{N}_{\varphi}$. We already mentioned that $\hat{\nabla}^{i t} \Lambda(x)=\Lambda\left(\tau_{t}(x) \delta^{-i t}\right)$ for all $x \in \mathscr{N}_{\varphi}$ and by definition we have $\nabla^{i t} \Lambda(x)=\Lambda\left(\sigma_{t}(x)\right)$ and $P^{i t} \Lambda(x)=v^{\frac{t}{2}} \Lambda\left(\tau_{t}(x)\right)$ for all $x \in \mathscr{N}_{\varphi}$. Because $\tau_{t}(\delta)=\delta$ for all $t \in \mathrm{R}$ it is easy to verify that $P^{i t} \Gamma(x)=$ $v^{\frac{t}{2}} \Gamma\left(\tau_{t}(x)\right)$ for all $x \in \mathscr{N}_{\psi}$ and by definition we have $\nabla^{i t} \Gamma(x)=\Gamma\left(\sigma_{t}^{\prime}(x)\right)$ for all $x \in \mathscr{N}_{\psi}$.

Using that all three one-parametergroups $\sigma, \sigma^{\prime}$ and $\tau$ commute and that $\sigma_{t}\left(\delta^{i s}\right)=\sigma_{t}^{\prime}\left(\delta^{i s}\right)=v^{i s t} \delta^{i s}$ and $\tau_{t}\left(\delta^{i s}\right)=\delta^{i s}$ for all $s, t \in \mathrm{R}$, it is straightforward to check the first equality in equation (12), equation (13) and equation (16) by applying the operators to an element $\Lambda(x)$ with $x \in \mathscr{N}_{\varphi}$. Using proposition 2.11 and the fact that $\sigma_{t} R=R \sigma_{-t}^{\prime}$ we can check the equalities $\hat{J} \nabla^{i t} \hat{J}=\nabla^{-i t}$ and $\hat{J} P^{i t} \hat{J}=P^{i t}$ on a vector $\Gamma(x)$ when $x \in \mathscr{N}_{\psi}$. This gives the first equalities of equations (14) and (15), and the rest of equation (14) follows from modular theory, because $v^{\frac{i}{4}} J$ is the modular conjugation of $\psi$ in the GNS-construction $(H, \iota, \Gamma)$. By the biduality theorem we also get $J \hat{\nabla} J=\hat{\nabla}$ and $J P J=P^{-1}$. Because for all $t \in \mathrm{R}$ we have $\hat{\nabla}^{i t}=P^{i t} J \delta^{i t} J$, we get $\hat{\nabla}^{i t}=P^{-i t} \delta^{-i t}$. This implies that $\hat{\nabla}^{i t} \Lambda(x)=v^{-\frac{t}{2}} \Lambda\left(\delta^{-i t} \tau_{-t}(x)\right)$ for all $x \in \mathscr{N}_{\varphi}$ and we can check then the second equality in equation (12) on a vector $\Lambda(x)$ with $x \in \mathscr{N}_{\varphi}$.

Because $R(x)=\hat{J} x^{*} \hat{J}$ for all $x \in M$ and $R(\delta)=\delta^{-1}$, we get the second equality of equation (15). Equations (17) and (18) follow because $P^{i s}, \nabla^{i s}$ and $\nabla^{i s}$ implement respectively $\tau_{s}, \sigma_{s}$ and $\sigma_{s}^{\prime}$ on $M$. Also $\hat{\nabla}^{i s}$ implements $\tau_{s}$ on $M$ and this gives the first equality of equation (19). Because we already saw that $\hat{\nabla}^{i s}=P^{-i s} \delta^{-i s}$ the second equality of equation (19) follows immediately from equation (17).

By the biduality theorem we can indeed perform the operation stated in the proposition, because $P^{i t} \hat{\Lambda}(y)=v^{-\frac{t}{2}} \hat{\Lambda}\left(\hat{\tau}_{t}(y)\right)$ for all $y \in \mathscr{N}_{\hat{\varphi}}$ and so in a sense $\hat{P}=P$.

Recall that we introduced the GNS-maps $\Lambda, \Gamma, \hat{\Lambda}$ and $\hat{\Gamma}$ for the weights $\varphi$, $\psi, \hat{\varphi}$ and $\hat{\psi}$ respectively. Using this we will define now three new multiplicative unitaries on $H \otimes H$ and relate them with our multiplicative unitary $W$ we used all the time. Recall that we already mentioned $V$ in section 1.

DEFINITION 2.14. Applying the von Neumann algebraic counterpart of theorem 3.16 in [8] (and its right invariant version) to $(M, \Delta)$ and $(\hat{M}, \hat{\Delta})$, 
one can define the unitaries $V, \hat{W}$ and $\hat{V}$ on $H \otimes H$ by the following formulas.

$$
\begin{aligned}
V(\Gamma(x) \otimes \Gamma(y)) & =(\Gamma \otimes \Gamma)(\Delta(x)(1 \otimes y)) \text { for all } x, y \in \mathscr{N}_{\psi} \\
\hat{W}^{*}(\hat{\Lambda}(x) \otimes \hat{\Lambda}(y)) & =(\hat{\Lambda} \otimes \hat{\Lambda})(\hat{\Delta}(y)(x \otimes 1)) \text { for all } x, y \in \mathscr{N}_{\hat{\varphi}} \\
\hat{V}(\hat{\Gamma}(x) \otimes \hat{\Gamma}(y)) & =(\hat{\Gamma} \otimes \hat{\Gamma})(\hat{\Delta}(x)(1 \otimes y)) \text { for all } x, y \in \mathscr{N}_{\hat{\psi}} .
\end{aligned}
$$

Observe that all the unitaries $W, V, \hat{W}$ and $\hat{V}$ satisfy the pentagonal equation. This could be checked directly, but it also follows from the pentagonal equation for $W$ and the formulas appearing in proposition 2.15. Almost by definition we have that $\Delta(x)=W^{*}(1 \otimes x) W=V(x \otimes 1) V^{*}$ for all $x \in M$ and $\hat{\Delta}(y)=\hat{W}^{*}(1 \otimes y) \hat{W}=\hat{V}(y \otimes 1) \hat{V}^{*}$ for all $y \in \hat{M}$.

The relation between all these multiplicative unitaries is given in the next proposition.

Proposition 2.15. We have the following formulas.

$$
\begin{aligned}
\hat{W} & =\Sigma W^{*} \Sigma \\
V & =(\hat{J} \otimes \hat{J}) \Sigma W^{*} \Sigma(\hat{J} \otimes \hat{J}) \\
\hat{V} & =(J \otimes J) W(J \otimes J) .
\end{aligned}
$$

So we have $\hat{W} \in \hat{M} \otimes M, V \in \hat{M}^{\prime} \otimes M$ and $\hat{V} \in M^{\prime} \otimes \hat{M}$.

Proof. The first equality follows from proposition 8.16 in [8]. Combining lemma 8.26 in [8] and our proposition 2.11 we get the second equality. Dualizing this we get $\hat{V}=(J \otimes J) \Sigma \hat{W}^{*} \Sigma(J \otimes J)$ and this gives the third equality after applying the first one.

The final statement follows from the fact that $W \in M \otimes \hat{M}$, the previous formulas and the equalities $J M J=M^{\prime}, \hat{J} \hat{M} \hat{J}=\hat{M}^{\prime}, \hat{J} M \hat{J}=M$ and $J \hat{M} J=\hat{M}$. The first two of these equalities follow from modular theory and the last two from proposition 2.1.

\section{A stronger form of left invariance}

In this section we want to prove some stronger form of left invariance of the Haar weight $\varphi$. We want to show that $(\iota \otimes \iota \otimes \varphi)(\iota \otimes \Delta)(X)=(\iota \otimes \varphi)(X) \otimes 1$ for any positive element $X \in N \otimes M$ and any von Neumann algebra $N$. The same formula is stated in [2] for Kac algebras, but not proved. The first proof for this formula in the Kac algebra case was given bij Zsidó in [19] (see also remark 18.23 in [12]). Unfortunately the proof of Zsidó does not work in the case of an arbitrary von Neumann algebraic quantum group, where possibly $\tau_{t} \neq \iota$. 
In our definition of a von Neumann algebraic quantum group we assumed the existence of invariant weights. The notion of left invariance we use, is in fact the weakest form of left invariance that one can assume, namely $\varphi((\omega \otimes$ ı) $\Delta(x))=\varphi(x) \omega(1)$ for all $\omega \in M_{*}^{+}$and $x \in \mathscr{M}_{\varphi}^{+}$. As a special case of the next proposition we will get the strongest form of left invariance, namely $(\iota \otimes$ $\varphi) \Delta(x)=\varphi(x) 1$ for all $x \in M^{+}$. Some result in between was already proved in proposition 5.15 of [8], and will be used in the proof of the proposition.

Proposition 3.1. Let $N$ be a von Neumann algebra and $X \in(N \otimes M)^{+}$. Then we have

$$
(\iota \otimes \iota \otimes \varphi)(\iota \otimes \Delta)(X)=(\iota \otimes \varphi)(X) \otimes 1
$$

Here both sides of the equation make sense in $(N \otimes M)_{\mathrm{Ext}}^{+}$. In particular we get

$$
(\iota \otimes \varphi) \Delta(x)=\varphi(x) 1
$$

for all $x \in M^{+}$.

Proof. We will prove the proposition for the dual von Neumann algebraic quantum group $(\hat{M}, \hat{\Delta})$. Because of the biduality theorem this proves the stated result. We also represent $N$ on a Hilbert space $K$ and then it is enough to prove the proposition in case $N=B(K)$.

Recall that we introduced the multiplicative unitary $\hat{V}$ in definition 2.14 . Then define for every $z \in B(K \otimes H)^{+}$the element $T(z) \in B(K \otimes H)_{\mathrm{Ext}}^{+}$by the following formula, which makes sense because $\hat{V} \in B(H) \otimes \hat{M}$.

$$
T(z)=(\iota \otimes \iota \otimes \hat{\varphi})\left((1 \otimes \hat{V})(z \otimes 1)\left(1 \otimes \hat{V}^{*}\right)\right) .
$$

When $\eta \in K \otimes H$ we denote by $P_{\eta}$ the positive rank one operator defined by $P_{\eta}(\xi)=\langle\xi, \eta\rangle \eta$. Let now $\eta \in K \otimes H$ and suppose $\|\eta\|=1$. Choose an orthonormal basis $\left(e_{i}\right)_{i \in I}$ of $K \otimes H$ such that $\eta=e_{i}$ for some $i \in I$.

Choose $\rho \in K \otimes H$. Then we have

$$
\begin{aligned}
& \left\langle T\left(P_{\eta}\right), \omega_{\rho}\right\rangle \\
& =\hat{\varphi}\left(\left(\omega_{\rho} \otimes \iota\right)\left((1 \otimes \hat{V})\left(P_{\eta} \otimes 1\right)\left(1 \otimes \hat{V}^{*}\right)\right)\right) \\
& =\sum_{i \in I} \hat{\varphi}\left(\left(\left(\omega_{\rho, e_{i}} \otimes \iota\right)\left(\left(P_{\eta} \otimes 1\right)\left(1 \otimes \hat{V}^{*}\right)\right)\right)^{*}\left(\omega_{\rho, e_{i}} \otimes \iota\right)\left(\left(P_{\eta} \otimes 1\right)\left(1 \otimes \hat{V}^{*}\right)\right)\right) \\
& =\hat{\varphi}\left(\left(\left(\omega_{\rho, \eta} \otimes \iota\right)\left(1 \otimes \hat{V}^{*}\right)\right)^{*}\left(\omega_{\rho, \eta} \otimes \iota\right)\left(1 \otimes \hat{V}^{*}\right)\right) .
\end{aligned}
$$

In proposition 2.15 we saw that $\hat{V}^{*}=(J \otimes J) W^{*}(J \otimes J)=\left(w^{*} \otimes 1\right) W(w \otimes 1)$ where $w=\hat{J} J$. The last equality follows from corollary 2.2. So it follows that $\left\langle T\left(P_{\eta}\right), \omega_{\rho}\right\rangle=\hat{\varphi}\left(\left(\left(\omega_{(1 \otimes w) \rho,(1 \otimes w) \eta} \otimes \iota\right)(1 \otimes W)\right)^{*}\left(\omega_{(1 \otimes w) \rho,(1 \otimes w) \eta} \otimes \iota\right)(1 \otimes W)\right)$. 
In remark 8.31 of [8] we saw that for $\omega \in M_{*}$ one has $(\omega \otimes \iota)(W) \in \mathscr{N}_{\hat{\varphi}}$ if and only if $\omega \in \mathscr{I}$. So it follows that $\left\langle T\left(P_{\eta}\right), \omega_{\rho}\right\rangle<\infty$ if and only if

$$
\omega_{(1 \otimes w) \rho,(1 \otimes w) \eta}(1 \otimes \cdot) \in \mathscr{I}
$$

and in that case

$$
\left\langle T\left(P_{\eta}\right), \omega_{\rho}\right\rangle=\left\|\xi\left(\omega_{(1 \otimes w) \rho,(1 \otimes w) \eta}(1 \otimes \cdot)\right)\right\|^{2} .
$$

Suppose that $u \in M$ is a unitary. And suppose that formula (20) is valid. We claim that

$$
\omega_{(1 \otimes w)(1 \otimes J u J) \rho,(1 \otimes w) \eta}(1 \otimes \cdot) \in \mathscr{I}
$$

and

$$
\xi\left(\omega_{(1 \otimes w)(1 \otimes J u J) \rho,(1 \otimes w) \eta}(1 \otimes \cdot)\right)=R\left(u^{*}\right) \xi\left(\omega_{(1 \otimes w) \rho,(1 \otimes w) \eta}(1 \otimes \cdot)\right) .
$$

For this choose $x \in \mathscr{N}_{\varphi}$ and make the following computation:

$$
\begin{aligned}
\omega_{(1 \otimes w)(1 \otimes J u J) \rho,(1 \otimes w) \eta}\left(1 \otimes x^{*}\right) & =\left\langle\left(1 \otimes x^{*}\right)(1 \otimes \hat{J} u J) \rho,(1 \otimes w) \eta\right\rangle \\
& =\left\langle\left(1 \otimes x^{*}\right)\left(1 \otimes R\left(u^{*}\right)\right)(1 \otimes w) \rho,(1 \otimes w) \eta\right\rangle \\
& =\left\langle\xi\left(\omega_{(1 \otimes w) \rho,(1 \otimes w) \eta}(1 \otimes \cdot)\right), R(u) \Lambda(x)\right\rangle \\
& =\left\langle R\left(u^{*}\right) \xi\left(\omega_{(1 \otimes w) \rho,(1 \otimes w) \eta}(1 \otimes \cdot)\right), \Lambda(x)\right\rangle
\end{aligned}
$$

From this follows our claim.

But then we get for every $\rho \in K \otimes H$ and every unitary $u \in M$ that

$$
\left\langle T\left(P_{\eta}\right), \omega_{\rho}\right\rangle=\left\langle T\left(P_{\eta}\right), \omega_{(1 \otimes J u J) \rho}\right\rangle .
$$

From this we may conclude that $T\left(P_{\eta}\right) \in(B(K) \otimes M)_{\mathrm{Ext}}^{+}$, for all $\eta \in K \otimes H$. Let now $z \in B(K \otimes H)^{+}$. Let $\left(e_{i}\right)_{i \in I}$ again be an orthonormal basis for $K \otimes H$. Then

$$
z=\sum_{i \in I} z^{1 / 2} P_{e_{i}} z^{1 / 2}=\sum_{i \in I} P_{z^{1 / 2} e_{i}} .
$$

By lower semicontinuity of $T$ we can conclude that $T(z) \in(B(K) \otimes M)_{\text {Ext }}^{+}$.

Let now $X \in(B(K) \otimes \hat{M})^{+}$. Then

$$
T(X)=(\iota \otimes \iota \otimes \hat{\varphi})(\iota \otimes \hat{\Delta})(X)
$$

and this clearly belongs to $(B(K) \otimes \hat{M})^{+}$Ext. But it also belongs to $(B(K) \otimes$ $M)_{\text {Ext }}^{+}$by the result in the previous paragraph. Let

$$
T(X)=\infty \cdot(1-e)+\int_{0}^{\infty} \lambda d e_{\lambda}
$$


be the unique spectral decomposition of $T(X)$, considered as an element of $B(K \otimes H)_{\text {Ext }}^{+}$. Then

$$
e, e_{\lambda} \in(B(K) \otimes M) \cap(B(K) \otimes \hat{M})=B(K) \otimes C
$$

because $M \cap \hat{M}=\mathrm{C}$. So take $f, f_{\lambda} \in B(K)$ such that $e=f \otimes 1$ and $e_{\lambda}=f_{\lambda} \otimes 1$. Then define the element $S \in B(K)_{\text {Ext }}^{+}$by

$$
S=\infty \cdot(1-f)+\int_{0}^{\infty} \lambda d f_{\lambda} .
$$

Then we get that

$$
(\iota \otimes \iota \otimes \hat{\varphi})(\iota \otimes \hat{\Delta})(X)=S \otimes 1
$$

Let us now suppose first that $K=\mathrm{C}$. This will prove the special case stated in the proposition. Then $X \in \hat{M}^{+}$and $S$ will be a scalar. So we get a $\lambda \in[0,+\infty]$ such that

$$
(\iota \otimes \hat{\varphi}) \hat{\Delta}(X)=\lambda 1
$$

Now there are two possibilities.

- Either there exists a $\omega \in \hat{M}_{*}^{+}$with $\omega \neq 0$ such that $(\omega \otimes \iota) \hat{\Delta}(X) \in \mathscr{M}_{\hat{\varphi}}^{+}$. Then $\lambda<+\infty$ because

$$
\lambda \omega(1)=\hat{\varphi}((\omega \otimes \iota) \hat{\Delta}(X))<\infty .
$$

But then also

$$
\hat{\varphi}((\mu \otimes \iota) \hat{\Delta}(X))=\lambda \mu(1)<\infty
$$

for all $\mu \in \hat{M}_{*}^{+}$, and so $(\mu \otimes \iota) \hat{\Delta}(X) \in \mathscr{M}_{\hat{\varphi}}^{+}$for all $\mu \in \hat{M}_{*}^{+}$. Then it follows from proposition 5.15 in [8] that $X \in \mathscr{M}_{\hat{\varphi}}^{+}$and so $\lambda=\hat{\varphi}(X)$ because of left invariance.

- Either we have $\hat{\varphi}((\omega \otimes \imath) \hat{\Delta}(X))=+\infty$ for all $\omega \in \hat{M}_{*}^{+} \backslash\{0\}$. This means that $\lambda=+\infty$. Because of left invariance we cannot have $X \in \mathscr{M}_{\hat{\varphi}}^{+}$and so $\hat{\varphi}(X)=+\infty$. Again $\lambda=\hat{\varphi}(X)$.

In both cases we arrive at $(\iota \otimes \hat{\varphi}) \hat{\Delta}(X)=\hat{\varphi}(X) 1$.

Now we return to the general case. Let $\omega \in B(K)_{*}^{+}$and $\mu \in \hat{M}_{*}^{+}$. Then we apply $\omega \otimes \mu$ to equation (21). This gives us

$$
\begin{aligned}
\langle S, \omega\rangle \mu(1) & =\hat{\varphi}((\omega \otimes \mu \otimes \iota)(\iota \otimes \hat{\Delta})(X))=\hat{\varphi}((\mu \otimes \iota) \hat{\Delta}((\omega \otimes \iota)(X))) \\
& =\mu(1) \hat{\varphi}((\omega \otimes \iota)(X))=\mu(1)\langle(\iota \otimes \hat{\varphi})(X), \omega\rangle .
\end{aligned}
$$


In this computation we used the special case of the proposition proved above. So it follows that $S=(\iota \otimes \hat{\varphi})(X)$ and this gives what we wanted to prove.

\section{The opposite and the commutant von Neumann algebraic quantum group}

Starting from $(M, \Delta)$, we can define two new von Neumann algebraic quantum groups called the opposite von Neumann algebraic quantum group $(M, \Delta)^{\text {op }}$ and the commutant von Neumann algebraic quantum group $(M, \Delta)^{\prime}$. With the notations introduced before we give the following definition.

Definition 4.1. The underlying von Neumann algebra of the opposite von Neumann algebraic quantum group $(M, \Delta)^{\text {op }}$ is again $M$ and the comultiplication $\Delta^{\mathrm{op}}$ is given by $\Delta^{\mathrm{op}}(x)=\chi \Delta(x)$ for all $x \in M$.

The underlying von Neumann algebra of the commutant von Neumann algebraic quantum group $(M, \Delta)^{\prime}$ is given by $M^{\prime}$ and the comultiplication $\Delta^{\prime}$ is defined by $\Delta^{\prime}(x)=(J \otimes J) \Delta(J x J)(J \otimes J)$ for all $x \in M^{\prime}$.

It is easy to see that $(M, \Delta)^{\mathrm{op}}$ and $(M, \Delta)^{\prime}$ are again von Neumann algebraic quantum groups. We will now give canonical choices for the left invariant weights and their GNS-construction. As a left invariant weight on $(M, \Delta)^{\text {op }}$ we take $\psi$, with GNS-construction $(H, \iota, \Gamma)$. On $M^{\prime}$ we define the weight $\varphi^{\prime}$ by $\varphi^{\prime}(x)=\varphi(J x J)$ for all $x \in\left(M^{\prime}\right)^{+}$. Then $\varphi^{\prime}$ is a left invariant weight on $(M, \Delta)^{\prime}$, with GNS-construction $\left(H, \iota, \Lambda^{\prime}\right)$, where $\Lambda^{\prime}(x)=J \Lambda(J x J)$ for all $x \in \mathscr{N}_{\varphi^{\prime}}$.

Given these GNS-constructions we can define the multiplicative unitaries $W^{\text {op }}$ and $W^{\prime}$ associated to $(M, \Delta)^{\mathrm{op}}$ and $(M, \Delta)^{\prime}$ and it is clear that, using definition 2.14 and proposition 2.15 , they are given by

$$
W^{\text {op }}=\Sigma V^{*} \Sigma \quad \text { and } \quad W^{\prime}=(J \otimes J) W(J \otimes J)=\hat{V} .
$$

It is also clear that the unitary antipode $R^{\mathrm{op}}$ of $(M, \Delta)^{\mathrm{op}}$ equals $R$ and the unitary antipode $R^{\prime}$ of $(M, \Delta)^{\prime}$ is given by $R^{\prime}(x)=J R(J x J) J$ for all $x \in M^{\prime}$. So the canonical right invariant weights on $(M, \Delta)^{\text {op }}$ and $(M, \Delta)^{\prime}$ are $\varphi$ and $\psi^{\prime}$. Then the modular elements $\delta^{\text {op }}$ and $\delta^{\prime}$ are given by

$$
\delta^{\mathrm{op}}=\delta^{-1} \quad \text { and } \quad \delta^{\prime}=J \delta J
$$

One also checks easily that $\tau_{t}^{\text {op }}$ equals $\tau_{-t}$ and $\tau_{t}^{\prime}(x)=J \tau_{-t}(J x J) J$ for all $t \in \mathrm{R}$ and $x \in M^{\prime}$.

Defining the unitary $w=\hat{J} J=v^{i / 4} J \hat{J}$ it is easy to see that $\Phi: M \rightarrow M^{\prime}$ : $\Phi(x)=w x w^{*}$ gives an isomorphism between the von Neumann algebraic quantum groups $(M, \Delta)$ and $(M, \Delta)^{\prime \text { op }}$. To prove this we only have to observe 
that $R(x)=\hat{J} x^{*} \hat{J}$ for all $x \in M$ and $(R \otimes R) \Delta(x)=\Delta^{\mathrm{op}}(R(x))$ for all $x \in M$.

We conclude this section with the following formulas.

Proposition 4.2. With the notations introduced above we have:

$$
\begin{aligned}
(M, \Delta)^{\mathrm{op} \wedge} & =(M, \Delta)^{\wedge \prime} \\
(M, \Delta)^{\prime \wedge} & =(M, \Delta)^{\wedge \mathrm{op}} \\
(M, \Delta)^{\prime \mathrm{op}} & =(M, \Delta)^{\mathrm{op} \prime} .
\end{aligned}
$$

Proof. Because $W^{\text {op }}=\Sigma V^{*} \Sigma$, the von Neumann algebra underlying $(M, \Delta)^{\mathrm{op} \wedge}$ is given by

$$
\left\{(\omega \otimes \iota)\left(W^{\mathrm{op}}\right) \mid \omega \in B(H)_{*}\right\}^{\prime \prime}=\left\{(\iota \otimes \omega)\left(V^{*}\right) \mid \omega \in B(H)_{*}\right\}^{\prime \prime}=\hat{M}^{\prime} .
$$

The last equality follows from proposition 2.15. Further we have for every $x \in \hat{M}^{\prime}$ that

$$
\Delta^{\mathrm{op} \wedge}(x)=\Sigma W^{\mathrm{op}}(x \otimes 1)\left(W^{\mathrm{op}}\right)^{*} \Sigma=V^{*}(1 \otimes x) V .
$$

Because $V=(\hat{J} \otimes \hat{J}) \Sigma W^{*} \Sigma(\hat{J} \otimes \hat{J})$, this gives

$$
\begin{aligned}
\Delta^{\mathrm{op} \wedge}(x) & =\Sigma(\hat{J} \otimes \hat{J}) W(\hat{J} x \hat{J} \otimes 1) W^{*}(\hat{J} \otimes \hat{J}) \Sigma \\
& =(\hat{J} \otimes \hat{J}) \hat{\Delta}(\hat{J} x \hat{J})(\hat{J} \otimes \hat{J})=\hat{\Delta}^{\prime}(x) .
\end{aligned}
$$

This gives $(M, \Delta)^{\mathrm{op} \wedge}=(M, \Delta)^{\wedge}$.

Applying this last formula to $(M, \Delta)^{\wedge}$ and using the biduality theorem we get $(M, \Delta)^{\wedge} \mathrm{op}^{\wedge}=(M, \Delta)^{\prime}$. Taking the dual and using once again the biduality theorem this gives our second result $(M, \Delta)^{\wedge} \mathrm{op}=(M, \Delta)^{\prime \wedge}$.

To compute $(M, \Delta)^{\mathrm{op} \prime}$ we have to observe once again that the modular conjugation $J^{\prime}$ of the left invariant weight $\psi$ on $(M, \Delta)^{\text {op }}$ is given by $J^{\prime}=$ $v^{i / 4} J$. Then it is clear that $(M, \Delta)^{\mathrm{op} \prime}=(M, \Delta)^{\prime \mathrm{op}}$.

\section{REFERENCES}

1. Baaj, S. and Skandalis, G., Unitaires multiplicatifs et dualité pour les produits croisés de $\mathrm{C}^{*}$-algèbres, Ann. Sci. École Norm. Sup. (4), 26 (1993), 425-488.

2. Enock, M. and Schwartz, J. -M., Produit croisé d'une algèbre de von Neumann par une algèbre de Kac II, Publ. Res. Inst. Math. Sci. 16 (1980), 189-232.

3. Enock, M. and Schwartz, J. -M., Kac Algebras and Duality of Locally Compact Groups, Springer-Verlag, Berlin (1992).

4. Haagerup, U., Operator valued weights in von Neumann algebras I, J. Funct. Anal. 32 (1979), 175-206. 
5. Kustermans, J., Induced corepresentations of locally compact quantum groups, J. Funct. Anal., to appear.

6. Kustermans, J., KMS-weights on $\mathrm{C}^{*}$-algebras, Preprint Odense Universitet (1997). \#functan/9704008

7. Kustermans, J., One-parameter representations on $\mathrm{C}^{*}$-algebras, Preprint Odense Universitet (1997). \#funct-an/9707010

8. Kustermans, J. and Vaes, S., Locally compact quantum groups, Ann. Sci. École Norm. Sup. (4) 33 (6) (2000), 837-934.

9. Kustermans, J. and Vaes, S., A simple definition for locally compact quantum groups, C. R. Acad. Sci., Paris Sér. I 328 (10) (1999), 871-876.

10. Kustermans, J. and Vaes, S., The operator algebra approach to quantum groups, Proc. Nat. Acad. Sci. USA 97 (2) (2000), 547-552.

11. Masuda, T. and Nakagami, Y., A von Neumann algebra framework for the duality of the quantum groups, Publ. Res. Inst. Math. Sci. 30 (1994), 799-850.

12. Stratila, S., Modular Theory in Operator Algebras, Abacus Press, Tunbridge Wells, England (1981).

13. Vaes, S., The unitary implementation of a locally compact quantum group action, J. Funct. Anal. 180 (2001), 426-480.

14. Vaes, S., A Radon-Nikodym theorem for von Neumann algebras, J. Operator Theory 46 (2001), 477-489.

15. Vainerman, L. I. and Kac, G. I., Nonunimodular ring-groups and Hopf-von Neumann algebras, Math. USSR-Sb. 23 (1974), 185-214.

16. Van Daele, A., An algebraic framework for group duality, Adv. Math. 140 (1998), 323-366.

17. Weil, A., L'intégration dans les groupes topologiques et ses applications, Actualités. Sci. Indust. 1145, (1953).

18. Woronowicz, S. L., From multiplicative unitaries to quantum groups, Internat. J. Math. 7 (1996), 127-149.

19. Zsidó, L., On the equality of two weights, Rev. Roumaine Math. Pures Appl. 23 (1978), 631-646.

DEPARTMENT OF MATHEMATICS

K. U. LEUVEN

CELESTIJNENLAAN 200 B

B-3001 LEUVEN

BELGIUM

E-mail: johan.kustermans@wis.kuleuven.ac.be
INSITUT DE MATHÉMATIQUES DE JUSSIEU ALGÈBRES D'OPÉRATEURS, PLATEAU 7E

175, RUE DE CHEVALERET

F-75013 PARIS

FRANCE

E-mail:vaes@math.jussieu.fr 